\title{
Intruding gravity currents and re-circulation in a rotating frame: Laboratory experiments
}

\author{
M. Eletta Negretti, ${ }^{1,}$ a) Francesco L. Tucciarone, ${ }^{1,2}$ and Achim Wirth ${ }^{1}$ \\ 1) Univ. Grenoble Alpes, CNRS, Grenoble INP, LEGI, 38000, Grenoble, France \\ ${ }^{2)}$ INRIA Rennes Bretagne Atlantique, IRMAR - UMR CNRS 6625, av. General Leclerc, 35042 Rennes, \\ France
}

(Dated: 8 October 2021)

\begin{abstract}
We present experimental results of rotating downslope gravity currents performed at the Coriolis Platform in Grenoble, France. A novel experimental design to produce the downslope gravity flow has been employed, using an axisymmetric configuration and a uniform flow injection that enabled to study the long term evolution of surface baroclinic vortices and of the gravity current, monitoring at the same time the evolution of the global circulation and the vorticity produced in the central deep area. The structure of the current, its relevant scales and the characteristics of the generated surface vortices fairly agree with previous results in the literature in smaller scale installations. Discrepancies are attributable to both the influence of topographic Rossby waves and viscous effects that are much reduced in the Coriolis Platform. Rotating intrusive gravity currents in a two-layer stratified ambient behave very differently from dense currents following the bottom slope. Substantial differences appear for the induced global circulation which depend on the nature of the intrusion, with a strong influence of the rotation rate. In particular, intruding gravity currents give rise to a strong turbulent environment at intermediate and bottom depths in the central area, with submesoscale vortices (i.e. with a typical size smaller than the Rossby deformation radius) and a large variety of scales. In contrast, when the dense current follows the bottom slope no significant vorticity production in the bottom and intermediate layers is reported. This clearly suggests that bottom boundary layers detaching from the boundary and propagating toward the ambient interior as in intrusive currents give an important contribution to the turbulence dynamics.
\end{abstract}

\section{INTRODUCTION}

Oceanic currents play a crucial role in the Earth's climate. They are driven by wind stress and subject to buoyancy forces, induced by variations in temperature and salinity, and influenced by the Coriolis force induced by the Earth's rotation. The abyssal waters are mainly fed by oceanic overflows, which are bottom-trapped dense gravity currents originating in semi-enclosed basins or on continental shelves ${ }^{1}$. Their descent on the continental slope involve boundary layers, high shears and density gradients, instabilities, breaking internal waves and the generation of sub-mesoscale vortices at [ $\mathrm{km}]$ scale and smaller. The resulting mixing influences the final stabilisation depth of the water mass, its density and transport, which controls the whole convection process. Examples of dense gravity currents are the Mediterranean outflow into the Atlantic Ocean ${ }^{2,3}$ and into the Black Sea ${ }^{4}$, the Ross Sea ${ }^{5}$ and the Denmark Strait overflow ${ }^{6}$. These flows involve processes that operate at spatial and temporal scales that cannot be captured by the resolution of global ocean circulation models and need to be correctly parametrized as reported for example in Danabasoglu, Large, and Briegleb ${ }^{7}$, Auclair et al. ${ }^{8}$. As discussed by Wells, Cenedese, and Caulfield ${ }^{9}$, the process of shear-driven entrainment associated with gravity currents is one of the key processes determining diapycnal transport in the world's oceans ${ }^{10-12}$. On a smaller scale, gravity currents related processes can produce interannual-to-decadal ocean variability as recently shown by Rubino et al. ${ }^{13}$.

Most of previous studies on gravity currents concentrated on dense currents (Simpson ${ }^{14}$, Britter and Linden ${ }^{15}$,

\footnotetext{
${ }^{a)}$ Electronic mail: eletta.negretti@legi.cnrs.fr
}

Cenedese and Adduce ${ }^{16}$, Ungarish ${ }^{17}$ and references herein) or intrusions in stratified ambients without background rotation $^{18-25}$ and often assuming self-similarity ${ }^{26}$. More recently Negretti, Flòr, and Hopfinger ${ }^{27}$ (see also ${ }^{28-32}$ ) considered the spatial development of currents over rapidly changing slopes for which self-similarity is not satisfied and reported important variations of the instability conditions and entrainment at the interface and on the bottom friction coefficient.

The dynamics of rotating gravity currents differs considerably from their non-rotating counter-parts ${ }^{33}$. So far, the effect of rotation has been explored in quasi-laminar regimes ${ }^{34-41}$. Mixing properties of a density driven current in a rotating frame were analyzed by Cenedese and Adduce ${ }^{42}$, De Falco et $a l .{ }^{43}$ as a function of topography, Reynolds and Froude numbers for different regimes. Laboratory experiments on eddying gravity currents were performed by Cenedese et al. ${ }^{44}$ who identified different regimes depending on the initial flow conditions (density anomaly, injection flow rate and rotation speed), exhibiting large-sale instabilities with the formation of either waves or domes in the dense current. In this latter regime, cyclonic vortices appear periodically in the fresh layer above the gravity current. These were already observed and analyzed by Lane-Serff and Baines ${ }^{38,39}$, Whitehead ${ }^{45}$, Etling et al. ${ }^{46}$, Decamp and Sommeria ${ }^{47}$. Their formation was either related to conserved potential vorticity in a water column stretching process ${ }^{48}$ or by growing baroclinic instability ${ }^{40}$. The eddies were predicted to travel along isobaths with a characteristic speed which depends on the reduced gravity, bottom slope and on the Coriolis parameter ${ }^{49}$.

In rotating gravity currents, bottom friction has usually been considered in the 'boundary layer' approximation ${ }^{45,50}$ and is valid only if the thickness of the dense layer sufficiently exceeds that of the Ekman boundary layer ${ }^{37,51}$, but the contri- 
bution of the bottom boundary layer turbulence to the production of submesoscale eddies remains largely unexplored.

Yankovsky and Legg ${ }^{52}$ performed non-hydrostatic numerical simulations, resolving submesoscale variability, and observed in both 2D and 3D simulations that the submesoscale range is dominated by submesoscale instabilities, particularly the symmetric instability. They observed that in both the investigated 2D and 3D cases, Ekman drainage leads to descent of the geostrophic jet, forming a highly dense alongslope front and beams of negative Ertel potential vorticity that initiate the submesoscale baroclinic instabilities with consequent vigorous mixing in the overflow. The evolution of a rotating gravity current descending a slope and detaching from the slope to intrude into a stratified ambient has been investigated little so $\mathrm{far}^{53}$.

Topographically generated sub-mesoscale instabilities have also been proposed as a new route for energy dissipation of geostrophic flows such as the overflow in the Labrador sea ${ }^{54}$, the Gulf Stream ${ }^{55}$ and the California Undercurrent ${ }^{56}$. Vic et al. ${ }^{57}$ and Akuetevi and Wirth ${ }^{58}$ found that sub-mesoscale vortices are generated by the roll-up of frictionally vortex filaments and engender dipoles that propagate balistically over distances several times their extension ${ }^{59}$.

While previous studies on rotating gravity currents considered one source to generate the gravity flow, mostly bounded in a channel, and concentrated on the generation of the surface baroclinic vortices ${ }^{35-38,44,46,47,60}$ or, more recently, on the topographic generated submesoscale vortices ${ }^{55,57,58}$, this article explores experimentally the long term evolution of the gravity current and of the baroclinic vortices generated by the gravity current at the surface, monitoring at the same time the subsequent evolution of the global circulation and vorticity produced in the central deep area. This was realized by performing experiments at the Coriolis Rotating Platform, the largest rotating facility in the world, allowing to approach the dynamical similarity for the main driving forces of buoyancy and Earth's rotation for ocean currents. In particular, a novel axisymmetric gravity current configuration enabled to create a undisturbed gravity current and thus to study the flow evolution over a very long duration (up to 220 rotational periods) and it allowed for azimuthal averages of the flow characteristics since time averages are limited due to the time evolution of the system. Another novelty consists in considering the intrusion of the gravity current into a two-layer stably stratified background in a rotating system, which mimics the conditions of the final state of oceanic overflows ${ }^{52,54}$, occurring in different areas of the World Ocean. This enabled also a better control of the vorticity that is generated by the saline intrusion in the central deep area with sustained turbulence production (no turbulence decay) and without imposing a characteristic length scale, to the contrary on previous studies that used moving grids ${ }^{61-63}$ or random operating flaps ejecting dipoles ${ }^{64}$. The present set-up gives a mechanism of turbulence production which is natural and closer to real ocean conditions.

The paper is organized as follows: section §II explains the experimental design, set-up and the measurements techniques. Inflow conditions for the gravity current and the characteristic flow scales are presented in section §III. Results relative to the induced surface baroclinic vortices are given in §IV and relative to the global circulation and the vorticity generated in the central deep area in $\S \mathrm{V}$ and $\S \mathrm{VI}$, respectively. §VII includes concluding remarks.

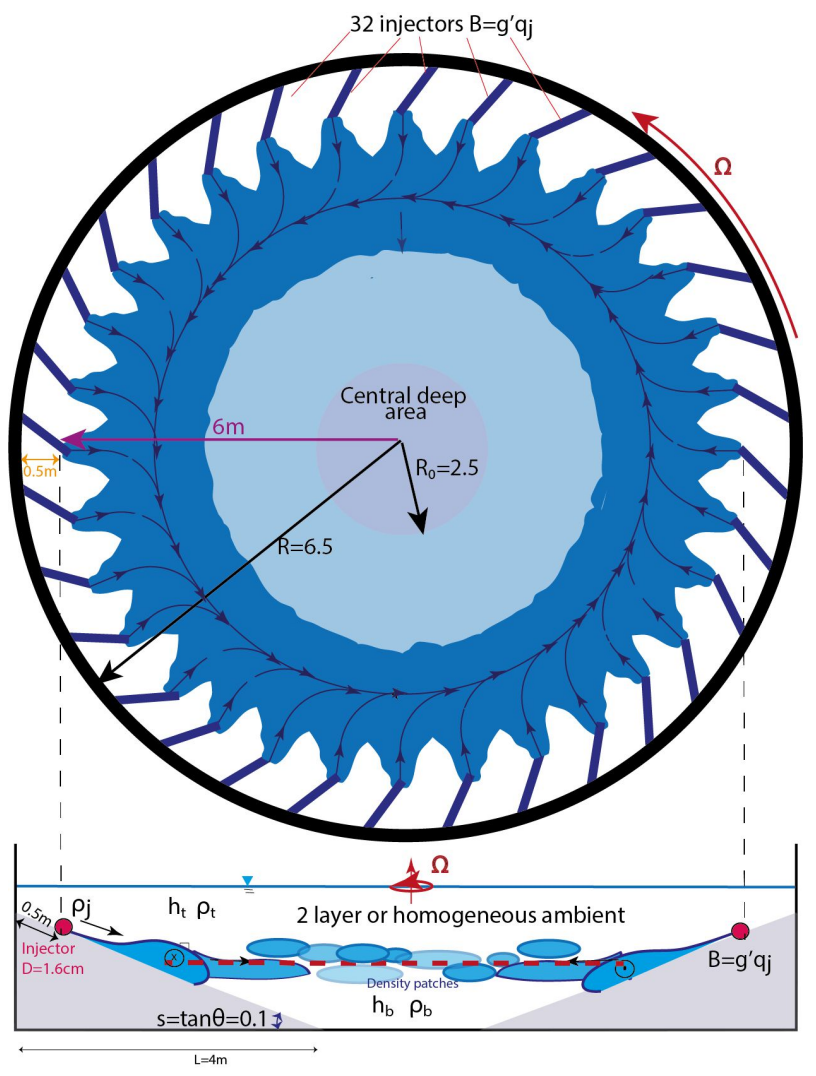

FIG. 1. Schematic top and side view of the experimental design. $N=32$ pipes positioned $50 \mathrm{~cm}$ from the tank edge and inclined by $45^{\circ}$ inject continuously saline solutions at a density of $\rho_{j}$ and with a constant flow rate ranging as $q_{j}=32-43 \mathrm{~cm}^{3} \mathrm{~s}^{-1}$ into a homogeneous or stably two-layer stratified ambient. The axisymmetric conical slope has an inclination of $6^{\circ}$ and a total length of $4 \mathrm{~m}$. The central deep area has a diameter of $5 \mathrm{~m}$.

\section{EXPERIMENTAL DESIGN}

The Coriolis Rotating Platform at LEGI (Grenoble, France) consists of a circular tank of $13 \mathrm{~m}$ diameter and $1.2 \mathrm{~m}$ depth $^{65}$. A sketch of the experimental design is given in figure 1 .

The tank was initially filled with two stably stratified discrete layers of different densities, $\rho_{t}$ and $\rho_{b}$, with $\rho_{b}>\rho_{t}$, and was put on anticlockwise (cyclonic) rotation. Particular attention was paid to the filling, which lasted several hours $(\approx 12$ hours) in order to ensure a sharp interface between the two layers and a solid body rotation with no residual motions.

Saline solutions with density $\rho_{j}$ were injected using a pump with a maximum capacity of $41 \mathrm{~s}^{-1}$ through $0.9 \mathrm{~cm}$ inner diameter plexiglass tubes at $N=32$ points equally spaced on the Coriolis platform's circumference at $r=6 \mathrm{~m}$ and inclined 
by $45^{\circ}$ in the sense of rotation to help geostrophic adjustment. The injection flow rate at each of the injectors was verified prior to each tank filling. The tubes were placed at the top of an inclined boundary, shaped as an inverted cone with a circular cross section descending toward the center of the tank (cf. figure 1). The inclined boundary descended from the tank edge starting with a height of $41 \mathrm{~cm}$ toward the center of the tank over a length of $4 \mathrm{~m}$ yielding a slope of $s=0.1$, and leaving the portion of the tank within a radius of $R_{0}=2.5 \mathrm{~m}$ from the center with a constant depth, that will be called hereafter the 'central deep area'. This configuration enabled the injected saline current to approach the geostrophic equilibrium within the tubes without mixing with the fresh ambient water during the critical adjustment phase. Indeed, the total injected flow rate $Q=\sum_{j=1}^{N} q_{j}$ was chosen in order to have an outlet velocity close to the Nof speed $v_{g}=g^{\prime} s / f^{49}$, where $g^{\prime}=g \Delta \rho / \rho_{t}$ is the reduced gravity, with $\Delta \rho=\rho_{j}-\rho_{t}, g$ the gravitational acceleration and $f=4 \pi / T$ the Coriolis parameter, and $T$ the rotation period of the Coriolis platform. No water was discharged during the experiments to avoid creation of spurious global vorticity. Hence, the total water depth $H$ in the tank increased during the experiment. The maximum increment was $20 \%$ of the initial depth $H_{0}$ over a typical experimental duration of $t / T=110$ to 220 rotational periods depending on the experiment. The increment of the total water depth related to the characteristic time scale $T$ is approximately $0.1 \%$.

The performed experiments along with their main characteristics are listed in table I. Based on the relative values of $\rho_{j}, \rho_{t}$ and $\rho_{b}$, three sets of experiments have been conducted and are depicted in figure 2. The first, called hereafter the 'homogeneous' case $H$, has $\rho_{j}>\rho_{t}=\rho_{b}$ and represents a dense gravity current, with the injected saline solutions reaching the bottom of the central deep area and no initial two-layer stratification in the ambient (cf. figure 2a). The second has $\rho_{t}<\rho_{j}<\rho_{b}$ and is hereafter called the 'intrusive' case $I$, with a thin tongue of injected density intruding at the pycnocline (cf. figure 2b) of the two-layer ambient stratification. The third, called the 'bottom' case $B$, has $\rho_{t}<\rho_{j} \approx \rho_{b}$ and the intrusion penetrated over the full depth of the bottom layer (cf. figure 2c).

In the following, we present results for specific reference experiments representative of these three cases: experiment $H$, $I 2$ for the intrusive cases and $B I$ for the bottom cases (see table table I). The reproducibility of the results has been verified analyzing the data of each of the experiments listed in table I.

\section{A. Measurement techniques}

The velocities were determined using the optical, nonintrusive experimental technique of Particle Image Velocimetry (PIV). The PIV set-up consisted of a light source, light sheet optics, seeding particles, a camera, and a PC equipped with a frame grabber and image acquisition software. Polyamide particles (Orgasol) with a mean diameter of $60 \mu \mathrm{m}$ and a specific density of $1.016 \mathrm{~kg} \mathrm{~m}^{-3}$ were added in the ambient water having density $\rho_{t}$ and $\rho_{b}$, respectively, and in the injected saline solutions of density $\rho_{j}$ as tracer material for the PIV measurements. A $25 \mathrm{~W}$ Yag laser operating at a wavelength $\lambda=532 \mathrm{~nm}$ provided a continuous light source. The beam was transmitted through a set of mirrors in the center of the tank where a high-speed rotating mirror positioned within a circular glass barrier with a diameter of $30 \mathrm{~cm}$ enabled to illuminate the full tank over an area of $130 \mathrm{~m}^{2}$. The system was allowed to move vertically along a Labview controlled linear axis to scan the water depth yielding 16 levels, not equally spaced (cf. figure 3), the highest and lowest levels being at $51 \mathrm{~cm}$ and $7 \mathrm{~cm}$ from the bottom, respectively and with distances ranging between the slices of $6 \mathrm{~cm}$ maximum at the top layers down to $1 \mathrm{~cm}$ in the critical pycnocline levels. Images of $13 \mathrm{~m} \times 9 \mathrm{~m}$ were taken with a high-resolution Nikon Camera (D850 SLR, 45MPx) synchronized with the laser system, at a frame rate of $1 \mathrm{~Hz}$, where three images were taken at each level. The time required to scan one volume consisting of the 16 levels was $t_{V}=86.5 \mathrm{~s}$. The spatial resolution of $1 \mathrm{~mm} /$ pixel was obtained using an optical lens (14 $\mathrm{mm}$ and F2.8) on the Nikon Camera.

Velocity fields were computed using a cross-correlation PIV algorithm encoded with the software UVMAT developed at LEGI. For this purpose, an adaptive multi-pass routine was used, starting with an interrogation window of $35 \times 35$ pixels and a final window size of $20 \times 20$ pixels, with a $70 \%$ window overlap. Each element of the resulting vector field thus represents an area of roughly $0.5 \times 0.5 \mathrm{~cm}$. The maximum experimental error is estimated to be about $3 \%$ in the instantaneous velocity and about $7 \%$ in its spatial derivatives.

The velocity in the injection region was monitored by means of an Acoustic Doppler Velocimetry Profiler (ADVP, Vectrino) in order determine the inlet conditions, i.e. the vein and Ekman layer characteristics. The ADVP is known to be more appropriate to resolve the current close to the bottom compared to the PIV technique. The seeding particles for PIV served also as the needed particulate for the ADV measurements. The instrument was mounted $1.1 \mathrm{~m}$ from the tank edge on a traversing system parallel to the bottom slope along a radial trajectory of $1 \mathrm{~m}$ length between two adjacent injectors (cf figure 3). Measurements were taken continuously throughout the experiment at $10 \mathrm{~cm}$ equally spaced positions for a total acquisition time of $60 \mathrm{~s}$ at $200 \mathrm{~Hz}$ for each position. The three velocity components $\left(u^{\prime}, v^{\prime}, w^{\prime}\right)$ on a coordinate system $\left(r^{\prime}, \theta, z^{\prime}\right)$ parallel and normal to the slope, were measured in a vertical section up to $z^{\prime}=3.2 \mathrm{~cm}$ from the bottom with measured points equally spaced by $0.2 \mathrm{~cm}$.

A 125 MicroScale Conductivity and Temperature Instrument (MSCTI, PME Vista, California, USA) was employed to measure the electrical conductivity and temperature of the water in the central deep area, yielding two analog outputs, one linearly proportional to the solution conductivity, and one non-linearly proportional to the solution temperature. The conductivity probe was installed at a distance of $1.08 \mathrm{~m}$ from the centre of the tank (see figure 3 ) mounted on a $1 \mathrm{~m}$ axis moving vertically throughout the full duration of the experiment with a velocity of $1 \mathrm{~cm} \mathrm{~s}^{-1}$ between $z=1$ to $51 \mathrm{~cm}$, with a resulting time between each vertical scan of $60 \mathrm{~s}$. The probe was calibrated at the beginning and at the end of each experiment to convert the voltage to density units $\mathrm{kg} \mathrm{m}^{-3}$. The 


\begin{tabular}{cccccccccc}
\hline EXP & $\begin{array}{c}h_{b}, \rho_{b} \\
{\left[\mathrm{~cm}_{\mathrm{kg} \mathrm{m}}{ }^{-3}\right]}\end{array}$ & $\begin{array}{c}h_{t}, \rho_{t} \\
{\left[\mathrm{~cm}^{2} \mathrm{~kg} \mathrm{~m}^{-3}\right]}\end{array}$ & $\begin{array}{c}\rho_{j} \\
{\left[\mathrm{~kg} \mathrm{~m}^{-3}\right]}\end{array}$ & $\begin{array}{c}g^{\prime} \\
{\left[\mathrm{cm} \mathrm{s}^{-2}\right]}\end{array}$ & $\begin{array}{c}f \\
{\left[\mathrm{~s}^{-1}\right]}\end{array}$ & $\begin{array}{c}\mathrm{Q} \\
{\left[\mathrm{s} \mathrm{s}^{-1}\right]}\end{array}$ & $\begin{array}{c}\mathscr{S} \\
{[-]}\end{array}$ & $\begin{array}{c}t_{\exp } \\
t / T\end{array}$ & $\begin{array}{c}\mathrm{n} \\
\Delta t_{1} \rightarrow \Delta t_{4}\end{array}$ \\
\hline$H$ & n.a.,n.a. & $53.9,999.1$ & 1005.5 & 6.28 & 0.14 & 1.36 & 3.01 & 146 & $8 \rightarrow 7$ \\
\hline$I 1$ & $22,1014.2$ & $31.9,999.4$ & 1004.3 & 4.8 & 0.14 & 1.37 & 4.0 & 146 & $8 \rightarrow 6$ \\
$I 2$ & $16,1013.4$ & $37.9,999.1$ & 1004.3 & 5.1 & 0.14 & 1.39 & 3.8 & 146 & $11 \rightarrow 9$ \\
$I 3$ & $14,1013.7$ & $40,999.3$ & 1004.3 & 5.0 & 0.14 & 1.3 & 3.6 & 210 & $11 \rightarrow 9$ \\
$I 4$ & $17,1014.2$ & $46.5,999.1$ & 1004.7 & 5.5 & 0.21 & 1.15 & 4.4 & 170 & $13 \rightarrow 10$ \\
$I 5$ & $10.5,1014.5$ & $45.2,999.1$ & 1005.3 & 6.09 & 0.21 & 1.19 & 4.1 & 170 & $10 \rightarrow 9$ \\
$I 6$ & $14,1014.3$ & $39.9,999.1$ & 1004.7 & 5.5 & 0.21 & 1.36 & 5.2 & 220 & $13 \rightarrow 6$ \\
$I 7$ & $16,1014.1$ & $37.9,999.1$ & 1004.3 & 5.1 & 0.105 & 1.35 & 2.8 & 110 & $8 \rightarrow 6$ \\
\hline$B 1$ & $16,1014.8$ & $37.9,1000.2$ & 1013.2 & 12.75 & 0.14 & 1.37 & 1.5 & 110 & $5 \rightarrow 3$ \\
$B 2$ & $16,1014.1$ & $37.9,999.4$ & 1012.9 & 13.0 & 0.21 & 1.33 & 2.13 & 220 & $6 \rightarrow 4$ \\
$B 3$ & 16,1015 & $38,999.1$ & 1013.2 & 13.8 & 0.105 & 1.33 & 1 & 110 & $5 \rightarrow 3$ \\
\hline
\end{tabular}

TABLE I. Experimental parameters and initial conditions. $n$ represents the number of vortices observed at the surface layers which varies between the beginning of the experiment $\Delta t_{1}$ and later times $\Delta t_{4}$ (see section IV).

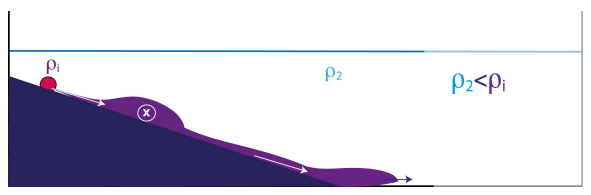

(a)

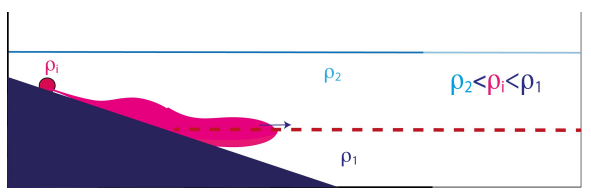

(b)

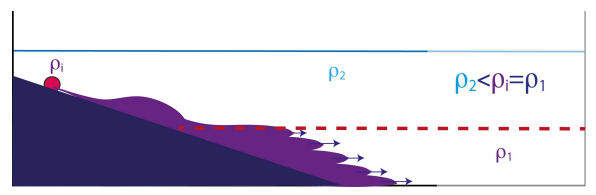

(c)

FIG. 2. Sketch of the three cases considered. (a) $H$ - Homogeneous case with no ambient stratification and an injection $\rho_{j}>\rho_{t}=\rho_{b}$; (b) $I$ Intrusive cases with $\rho_{t}<\rho_{j}<\rho_{b}$ and a tongue of saline water intrusion at the pycnocline; (c) $B$ - Bottom cases with $\rho_{t}<\rho_{j}=\rho_{b}$ and where the intrusion takes the form of fingers into the bottom layer.

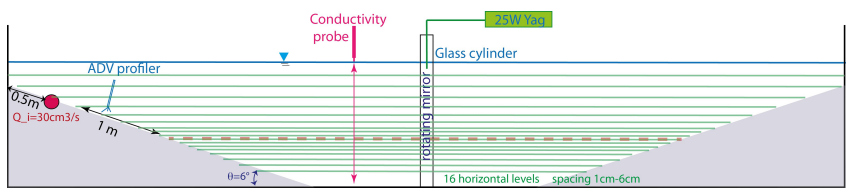

FIG. 3. Side view sketch of the experimental set-up with 16 horizontal slices for the PIV measurements, along with the ADV and conductivity probe positions mounted on a $1 \mathrm{~m}$ scanning axe.

density at the injection was constantly monitored by a fixed conductivity probe and was kept constant throughout the experiment.

Finally, flow visualization was realized using Rhodamine $6 \mathrm{G}$ at the injection to highlight the intruding current. The dye was injected at different times during the experiment and a GoPro camera equipped with an orange filter enabled to separate the Rhodamine emitted spectrum from that of the PIV particles.

\section{FLOW SCALES}

Once the flow rate is switched on, heavier fluid with respect to the ambient starts to exit the pipes and a gravity current is generated. With the given inclination of the pipes, the current is quickly deviated to the right subject to a geostrophic adjustment, resulting in a main velocity component in the azimuthal direction. Wirth ${ }^{33}$ demonstrated that the current has a dual structure consisting of a thick vein, governed by geostrophy and with an Ekman layer at the bottom, and a thin friction layer at the downslope side of the vein. For a detailed description of the structure of a rotating downslope gravity current it is referred to Wirth ${ }^{33}$ and references herein.

As the flow develops on the slope, it reaches the central deep area as an intrusion (two-layer stratified cases) or filling the bottom (homegeneous case), and eventually produces vortices of different size, of the order of the Rossby deformation radius and smaller.

\section{A. Gravity current scales}

An estimation of the characteristic scales of the gravity current on the slope can be obtained considering that the velocity $U$ must scale with $\sqrt{g^{\prime} h}$, where $h$ is the depth of the current; with the hypothesis of geostrophy also $f U=g^{\prime} h / L_{g}$ must hold, where $L_{g}$ represents a scale for the width of the gravity current. Combining these relations, the scales for the velocity $U$, the width $L_{g}$ and the height $h$ of the current can be obtained $^{39,47}$

$$
U=(B f)^{1 / 4}, \quad L_{g}=\left(B f^{-3}\right)^{1 / 4}, \quad h=(B f)^{1 / 2} / g^{\prime},
$$

where $B=g^{\prime} Q$ is the imposed buoyancy flux. Note also that combining the relations for $L_{g}$ and $h$ in equation 1, the expression for $L_{g}$ coincides with the definition of a Rossby deformation radius based on the depth of the current $h$.

As pointed out in Wirth ${ }^{33}$, friction (i.e. viscous effects) plays a key role in the evolution of rotating downslope gravity currents. In our experiments, the Ekman number is $E k=$ 


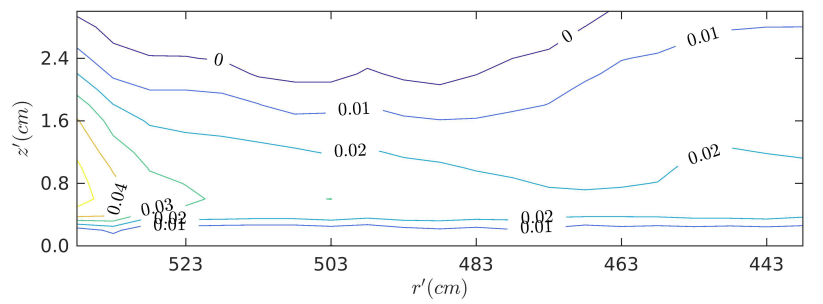

(a)

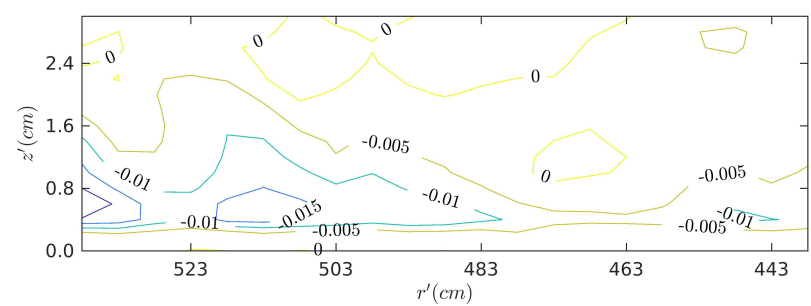

(b)

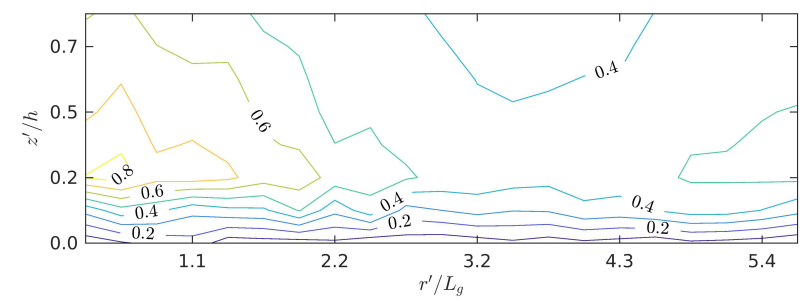

(c)

FIG. 4. Time averaged velocity contour plots for experiment $B 1$ over $t / T=50$ for the along slope $v^{\prime}$ (a) and cross slope $u^{\prime}$ (b) velocity components. Isolines give the velocity in $0.1 \mathrm{~m} \mathrm{~s}^{-1}$. (c) Contour plot of the norm of the velocity $u_{H}$ averaged over $t / T=50$ recorded with the ADVP and rescaled with the quantities of equation (1).

$2 v /\left(f H^{2}\right)=\mathscr{O}\left(10^{-2}\right)$, where $H$ is a vertical scale characterizing the typical water depth. Water from the vein detrains due to friction in the bottom Ekman layer $\delta_{E}=\sqrt{2 v / f} \approx 0.45 \mathrm{~cm}$ into the friction layer $\delta_{f} \approx 2 \delta_{E}{ }^{37}$. The estimated Ekman drainage (laminar) is $q_{E} \approx v_{g} \delta_{E}=\mathscr{O}\left(1 \mathrm{~cm}^{2} \mathrm{~s}^{-1}\right)$. The ratio between the flux that can be transported downslope by the Ekman dynamics and the injected flux is the flux Ekman number $D_{E k}$ that has been defined in Wirth and Negretti ${ }^{66}$. In the present experiments this ratio is always larger or equal (for experiment $I 6$ only) than unity.

The scalings for the gravity current are verified using the ADV velocity data captured $1.1 \mathrm{~m}$ from the tank edge (cf. figure 3). Time averaged velocity contour plots relative to $B 1$ over $t / T=50$ are shown in figure 4 for the two velocity components $v^{\prime}$ (a) and $u^{\prime}$ (b). Note that for these data the vertical and radial axis are normal and parallel to the slope, respectively. For the along slope velocity component $v^{\prime}$, part of the vein can be clearly distinguished in the region $r^{\prime}>520 \mathrm{~cm}$ and $0<z^{\prime}<2.5 \mathrm{~cm}$, with $v^{\prime}$ being the dominant velocity with maximum values of $5 \mathrm{~cm} \mathrm{~s}^{-1}$. The radial velocity $u^{\prime}$ (negative when descending the slope) is predominant in the thin layer $0<z^{\prime}<0.8-0.9 \mathrm{~cm}$ over the measured radial coordinate, which is in good accord with the prediction of the friction layer $\delta_{f} \approx 0.9 \mathrm{~cm}$.
Figure $4 \mathrm{c}$ displays the norm of the velocity recorded with the ADVP rescaled with the quantities given in equation 1 , showing that the normalized horizontal velocity $u_{H} / U \leq 0.8$ for $z^{\prime} / h<0.9$ and for $x^{\prime} / L_{g}=1$, with $u_{H}=\sqrt{u^{\prime 2}+v^{\prime 2}}$. Hence, the characteristic scales of the gravity current can be estimated using the relations proposed by Lane-Serff and Baines ${ }^{39}$ and given in equation 1. These scalings will be used in section IV to evaluate the characteristics of surface vortices generated by the gravity current and to compare the results to previous studies of rotating downslope gravity currents generated from a single source ${ }^{38,39,47}$.

\section{B. Overall scalings}

The dynamic of the system depends on the following independent variables $\left(s, L, h, g^{\prime}, f, v\right)$, where $L$ is a characteristic geometrical horizontal scale representative for the experimental tank, $h$ is a vertical scale for the current and $v$ is the kinematic viscosity of the fluid.

The overall relevant non-dimensional numbers are the Rossby number $R o=U / f L=\mathscr{O}\left(10^{-1}\right)$, the Reynolds number $R e=U L / v=\mathscr{O}\left(10^{6}\right)$ and the Burger number $B u=\mathscr{R}^{2} / L^{2}=$ $R o / F r=\mathscr{O}\left(10^{-1}-10^{-2}\right)$, with $\mathscr{R}=\sqrt{g^{\prime} h_{*}} / f$ the Rossby deformation radius defined based on the depth $h_{*}$ above the current (cf. Lane-Serff and Baines ${ }^{39}$ ), and $F r=U / \sqrt{g^{\prime} h}$ the Froude number. For the above estimations, typical values of $\mathscr{R} \sim \mathscr{O}(1 \mathrm{~m}), U \approx 0.1 \mathrm{~m} \mathrm{~s}^{-1}$ and $L \sim \mathscr{O}(10 \mathrm{~m})$ have been considered.

The buoyancy Reynolds number can be estimated as $R e_{b}=$ $R e L_{b}^{2} / L_{v}^{2}$, where $L_{b}$ and $L_{v}$ are buoyancy and viscous length scales, respectively. We estimate for the experiments $L_{b} \sim$ $0.3 f / N \mathscr{R} \approx 10 \mathrm{~cm}$ (Hedstrom and Armi ${ }^{67}$ ), with the Brunt Väisälaa frequency $N=\mathscr{O}(0.1) \mathrm{s}^{-1}$ deduced from the density measurements, and $L_{v} \approx \delta_{E}=0.45 \mathrm{~cm}$, leaving $\mathscr{R} e_{b}=$ $\mathscr{O}\left(10^{8}\right)$.

Comparing the injection velocity $u_{j}$ to the outlet of the injectors with the Nof speed $v_{g}$ gives the non-dimensional parameter $\mathscr{S}=u_{j} / v_{g}$. For $\mathscr{S}=1$ there is a balance between the gravity and the Coriolis force. In this case the gravity current has only the azimuthal velocity component corresponding to $v_{g}$ and would turn around the circumference with a constant radius on the slope infinitely, as long as mixing, viscous (frictional) effects are neglected (cf. figure 1 and Wirth ${ }^{33}$ ). When $\mathscr{S}>1$, a rather 'slow' current with respect to the background rotation is achieved, whereas for $\mathscr{S}<1$ we have a 'fast' current. In the present experiments this ratio always falls in the first case of a 'slow' current with $\mathscr{S}>1$.

\section{BAROCLINIC SURFACE VORTICES}

Previous studies ${ }^{38,40,46,68,69}$ have reported the formation of cyclonic vortices in the ambient water above the current. They inferred their formation to two main processes: the geostrophic adjustment process and the Ekman drainage. In the first case, if the fluid above the current is not moving with respect to the current layer, the upper layer is captured 


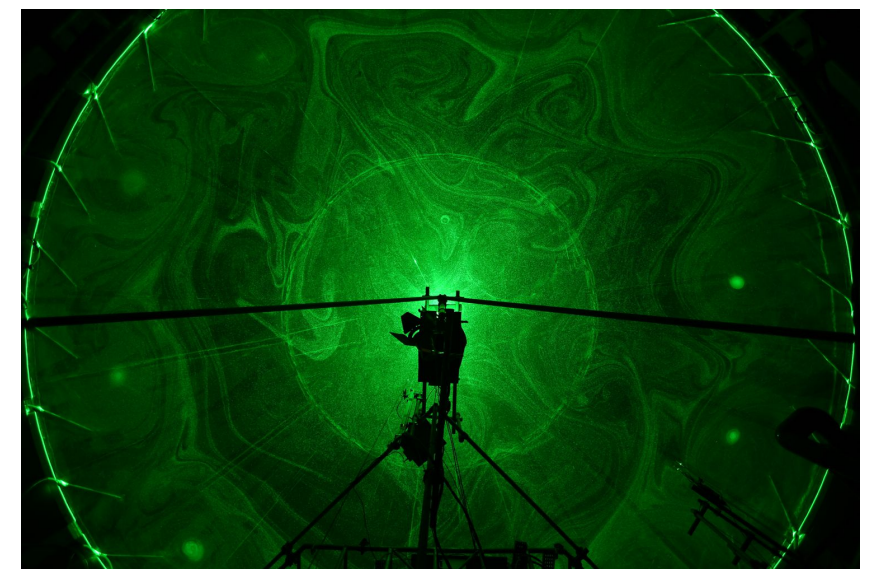

FIG. 5. Instantaneous image of the PIV field of view at the top layer $z=49 \mathrm{~cm}$ at $t / T=10$ with cyclonic vortices in the outer area evident from particles concentrated in their core. The horizontal scale of the image is $13 \mathrm{~m}$, the vertical scale $9 \mathrm{~m}$. The spatial resolution of the $\mathrm{PIV}$ is $1 \mathrm{~mm} /$ pixel.

by the downward current following it like a Taylor column. Using the thermal wind relation and the conservation of potential vorticity Lane-Serff and Baines ${ }^{38}$ derived a vorticity in the upper layer with the sign varying with $f$, thus cyclonic, due to stretching of the water column. In the second case, the cyclonic vorticity is induced in the upper layer by Ekman drainage due to entrainment into the downslope flowing friction layer, which also produces a stretching of the upper layer. The former mechanism occurs on a short time scale while the latter is a process that acts during the whole experiment; both concur to reduce the thickness of the gravity current.

After the initial adjustment phase lasting $t / T \sim 10$ the surface cyclonic vortices have formed and are organized in a circle of $r \approx 5.5 \mathrm{~m}$, as displayed in figure 5, that shows a top view of a raw PIV image of experiment $I 2$. The core of the vorticies is highlighted by white spots of concentrated PIV particles. These vortices persist until the end of the experiment, for all experiments performed.

The following sections will present their averaged characteristics. The temporal behaviour and a more thorough statistical analysis is investigated in detail elsewhere ${ }^{70}$.

\section{A. Averaged quantities}

To evaluate the averaged characteristics of the vortices, we focused on each of the vortices $n$ for each of the experiments listed in table I and the values has been averaged over those obtained for each of the first three surface levels. The chosen vortex has been tracked at different instants during the experiment representing a specific stage: one after the initial adjustment phase $\Delta t_{1}:$ from $t / T=0$ to $t / T \approx 10-20$, a second and third during the quasi-steady phase of the experiment $\Delta t_{2}:$ from $t / T \approx 10-20$ to $t / T \approx 55-110, \Delta t_{3}$ : from $t / T \approx 55-110$ to $t / T \approx 100-200$ and a latter $\Delta t_{4}:$ from $t / T \approx 100-200$ to $t / T=110-220$ for the final stage, in which a smooth decline of the vortices has been reported. The characteristics that have been evaluated are: the number of vortices $n$, their size $\mathscr{R}_{o b s}$ and phase speed $c_{o b s}$, which changed significantly from experiment to experiment.

The size of the vortices $\mathscr{R}_{o b s}$ has been evaluated approximating the vortex itself with a circle, centered in the core of the vortex, defined as the point of maximum vorticity $\zeta_{m}$, and incorporating the best fit of the positive (cyclonic) vorticity. To verify that the obtained value of $\mathscr{R}_{o b s}$ is not dependent on the specific method chosen, we compared these results to those obtained estimating their size from the transverse cut across the centre of the vortex (vorticity maximum) of the azimuthal velocity component. Determining the minimum and maximum values of this velocity transect, and taking the half width between the two extremes, gave a second estimation of $\mathscr{R}_{\text {obs }}$. The difference between these two methods in the estimation of $\mathscr{R}_{\text {obs }}$ was $7 \%$ maximum.

The phase speed $c_{o b s}$ has been estimated tracking the chosen vortex and making an average of its displacement $\Delta(r d \theta)$ over $m=5$ volume scans each separated by $t_{V}=86.5 \mathrm{~s}$. This procedure was repeated for each of the four considered characteristic times $\Delta t_{1}$ to $\Delta t_{4}$, i.e.

$$
\left[c_{o b s, i}=\frac{\sum_{i=1}^{m} \Delta(r d \theta)}{m t_{V}}\right]_{\Delta t_{i}}
$$

The estimated averaged characteristics are presented in figure 6 for all the experiments listed in table I, where the error bars are representative for the variability in time (over the four selected experiment stages $\Delta t_{1}$ to $\Delta t_{4}$ ), in space (between the first three horizontal levels) and between the $n$ vortices for each experiment.

The evaluated Rossby radius $\mathscr{R}_{o b s}$ of our experiments are given in figure 6a compared to the theoretical Rossby deformation radius $\mathscr{R}_{t h}=\sqrt{g^{\prime} h_{*}} / f$ (solid red line). The relation proposed by Lane-Serff and Baines ${ }^{38}$ and that proposed by Decamp and Sommeria ${ }^{47}$

$$
\mathscr{R}_{\text {obs }}=C \mathscr{R}_{t h}
$$

with the constant $C$ being $1.25 \pm 0.05$ and $0.88 \pm 0.06$, respectively, are given as well by the blue and grey shaded regions in figure 6 a. Since the study of Lane-Serff and Baines ${ }^{38}$ was realized in a much smaller installation, with the maximum size of the vortices being one order of magnitude less than the values reported in the present study, this relation do not fit our experimental data. Our data agree better with the relation proposed by Decamp and Sommeria ${ }^{47}$, which however still overestimates the reported values.

Lane-Serff and Baines ${ }^{38}$ also defined an important length scale to measure the viscous drainage, i.e.

$$
Y=\frac{Q}{v_{g} \delta_{E}}=\frac{Q f^{3 / 2}}{g^{\prime} s \sqrt{2 v}}
$$

which gives the length needed for the current to flow entirely in the viscous layer with the Nof speed. Small values of $Y$ represent strong viscous effects. Note that the length scale $Y$ 


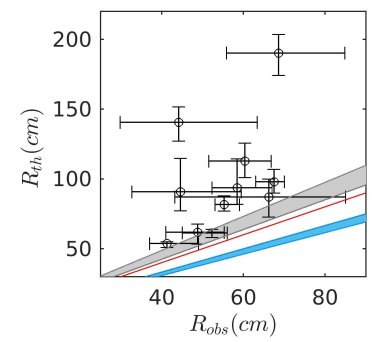

(a)

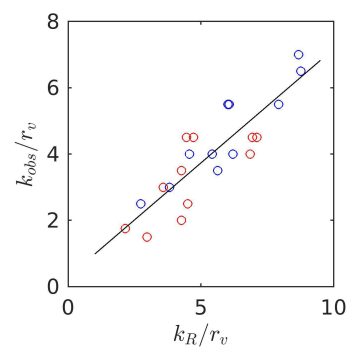

(c)

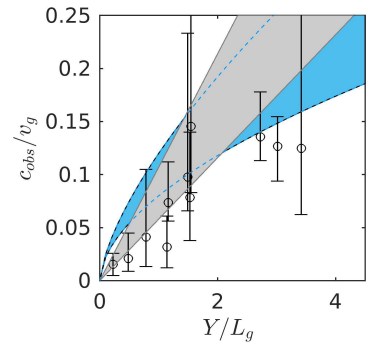

(b)

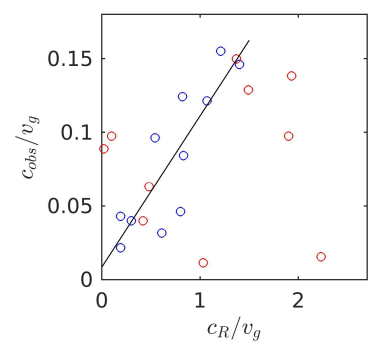

(d)
FIG. 6. (a) Theoretical Rossby deformation radius $\mathscr{R}_{t h}$ versus the observed radius $\mathscr{R}_{\text {obs }}$. (b) Normalized phase speed $c_{o b s} / v_{g}$ versus the viscous drainage $Y / L_{g}$. The blue and grey shaded regions in $(\mathrm{a}, \mathrm{b})$ represent the relations proposed by Lane-Serff and Baines ${ }^{38}$ and Decamp and Sommeria ${ }^{47}$, respectively (see equations (3) and (6)). (c) The observed normalized wavenumber $k_{o b s} / r_{v}$ of the surface vortices plotted versus the equilibrium wavenumber of topographic Rossby waves $k_{R} / r_{v}$ for all performed experiments and (d) the relative phase speeds $c_{o b s} / v_{g}$ and $c_{R} / v_{g}$. In (c,d), blue and red symbols represent the values averaged during the initial and final stages of the experiments, respectively. Black solid lines represent a linear fit over the full data: $k_{o b s} / r_{v}=0.69 k_{R} / r_{v}+0.3$ in (c) and over the initial data only (blue symbols): $c_{o b s} / v_{g}=0.1 c_{R} / v_{g}+0.008$ in (d).

can be extrapolated from the flux Ekman number $D_{E k}$ defined in §III B by imposing the ratio equal to unity.

The normalized phase speeds $c_{o b s} / v_{g}$ are reported as symbols in figure $6 \mathrm{~b}$ for the different experiments versus the viscous drainage scale $Y / L_{g}$. In general, we see that the phase speed of the vortices increases with larger values of the normalized viscous drainage scale $Y / L_{g}$ although there is a large variability in the data. For the normalized vortex speed LaneSerff and Baines ${ }^{38}$ and Decamp and Sommeria ${ }^{47}$ proposed, respectively, the relations

$$
\begin{aligned}
c / v_{g} & =(0.1 \pm 0.02)\left(Y / L_{g}\right)^{0.62 \pm 0.06} \\
c & =(0.28 \pm 0.03) U
\end{aligned}
$$

which are highlighted by the blue and grey shaded regions, respectively, in figure $6 \mathrm{~b}$. Our values lie slightly below both predictions.

One significant difference in the present study is the distributed axisymmetric injection, while in previous studies the injection was realized through a single source. One aspect that has not been discussed in those studies is the eventual presence of topographic Rossby waves, induced by the sloping boundary, which represents a topographic $\beta$-plane. Once the surface vortices have formed in the experiments, a wavenum- ber $k_{o b s}$ can be estimated using the total number of vortices observed, $n$, developed along the circumference $2 \pi r_{v}$, where $r_{v}$ is the radial distance from the centre of the tank to the core of the vortices (cf. table I and figure 5). This wavenumber can be compared to the equilibrium wavenumber of the topographic Rossby wave: $k_{R}=\sqrt{v_{g} \beta^{-1}}$. Herein, we used the geostrophic velocity $v_{g}$ as velocity scale and $\beta=s f / h_{*}$. Results are plotted in figure 6c. The normalized observed wavenumber $k_{o b s} / r_{v}$ is reported versus the normalized estimated topographic Rossby wavelength $k_{R} / r_{v}$. The blue symbols represent the averaged values in the first stage of the experiments $\left(\Delta t_{1}\right)$ (where the water depth $h_{*}$ is smaller resulting in a larger $\beta$ value and where there is a larger number of vortices $n$, cf. table I), while the red symbols represent values averaged over the final stage of the experiment $\left(\Delta t_{4}\right)$. The values collapse on the linear relation $k_{o b s} / r_{v}=0.69 k_{r} / r_{v}+0.3$. We can also estimate the speed $c_{R}=v_{g}-\beta / k_{o b s}^{2}$ using the observed wavenumber and compare these values to the speeds $c_{o b s}$ evaluated from the experiments (cf. equation 2). This is shown in figure $6 \mathrm{~d}$, where symbols are as given in figure 6c. There is a linear correlation between the phase speed of the topographic Rossby wave and the phase speed of the vortices determined from the experiments when considering the initial stage of the experiment, as given by the solid line: $c_{o b s} / v_{g}=0.1 c_{R} / v_{g}+0.008$, whereas data become sparse at later stages entering the decline phase of the experiment.

This suggests that in the present study, the size of the surface vortices and their phase speed is not only determined by the Rossby deformation radius, the viscous drainage $Y / L_{g}$ or the velocity $U$ as suggested by Lane-Serff and Baines ${ }^{39}$ and Decamp and Sommeria ${ }^{47}$, respectively, but they are also influenced by the topographic Rossby wave, explaining why our results in figures $6(a, b)$ do not fit with the observations of previous studies. Probably, in Lane-Serff and Baines ${ }^{39}$ and Decamp and Sommeria ${ }^{47}$ the effect of the topographic $\beta$-plane was negligible.

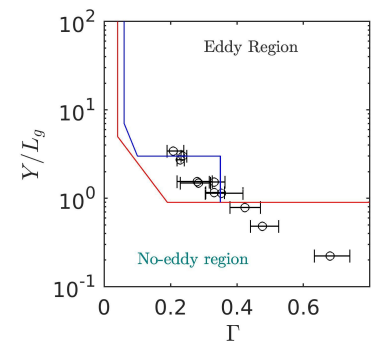

(a)

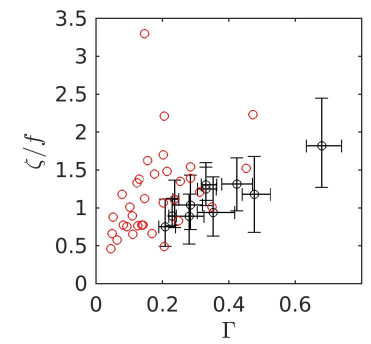

(b)
FIG. 7. (a) Viscous drainage $Y / L_{g}$ versus the stretching parameter $\Gamma$. The blue and red continuous lines represent the limit between the eddies formation region and the no-eddies region, along with the overlap region, as proposed by Lane-Serff and Baines ${ }^{38}$; (b) normalized vorticity $\zeta / f$ versus the stretching parameter $\Gamma$ for our experiments (black symbols) and those of Lane-Serff and Baines ${ }^{38}$ (red circles).

Lane-Serff and Baines ${ }^{38}$ argued that the formation of the baroclinic vortices in the upper layer is favored when the depth in the upper ambient layer is reduced, and related this to a vortex stretching mechanism for which the water column 
is sucked by the current of typical width $L_{g}$ over a depth of order $s L_{g}$. They defined as stretching parameter the ratio between this distance and the total depth above the current in the upper ambient water $h_{*}$, i.e. $\Gamma=s L_{g} / h_{*}$.

Figure $7 \mathrm{a}$ gives the relative influence of the stretching parameter $\Gamma$ and the viscous drainage $Y / L_{g}$, that has been classified into three areas by Lane-Serff and Baines ${ }^{38,39}$ based on their experimental data: the so called 'eddy region' above the blue line, a region without eddies, below the red line and an overlap region between these two areas that represents a transition region with possible formation of vortices.

Most of our experiments lay in the overlap and in the 'no eddy' regions, as reported in figure 7a. However, in our experiments the baroclinic vortices are clearly developed in all cases. Beside the effect of the topographic $\beta$-plane which may sustain the formation of the surface vortices in our case, the small-scale installation in Lane-Serff and Baines ${ }^{38,39}$ may have dampened the formation of such vortices with relevant viscous drainage.

The eddies form as a result of the stretching of fluid columns in the overlying fluid, which Lane-Serff and Baines ${ }^{38,39}$ explained to be produced by three processes. Two of these are due to the initial adjustment of the flow. The third process depends on stretching of the fluid column by Ekman drainage from below and operates continuously. As discussed in Lane-Serff and Baines ${ }^{38,39}$, this process causes a gradual downslope displacement of the inviscid current (the vein) with alongslope distance because of the larger Ekman drainage at the upslope side of the current. These processes are collectively represented by the parameters $\Gamma$ (the stretching parameter) and $Y / L_{g}$ (the viscous drainage scale). However, to compare between different laboratory experiments and then extrapolate the results to oceanographic situations, the similarity between the laboratory experiments and the oceanographic scenarios should be approached in terms of the turbulent eddy dissipation as well. For this, the molecular viscosity $v$ has to be replaced by the vertical eddy viscosity $v_{t}$. The Coriolis platform permits for a large range of scales, from $[10 \mathrm{~m}]$ down to the $[\mathrm{mm}]$ scale, i.e. four orders of magnitude.

Considering the gravity current in the ambient flow as a stratified shear layer, we can use the model proposed by J., Odier, and Ecke ${ }^{71}$ based on the Prandtl mixing length model with a characteristic mixing length $L_{m}$, to relate the turbulent eddy viscosity $v_{t}$ to the velocity fluctuations and the gradients in a stratified shear layer. They showed that $L_{m}$ was proportional to the shear scale $L_{s}=\left(\varepsilon /\left\langle\partial_{z} u\right\rangle^{3}\right)^{1 / 2}$ so that the turbulent eddy viscosity can be evaluated using

$$
v_{t} \sim L_{s}^{2}\left\langle\partial_{z} u\right\rangle
$$

with $\varepsilon$ being the dissipation rate in $\left[\mathrm{m}^{2} \mathrm{~s}^{-3}\right]$ and $\partial_{z} u$ is the vertical shear. In the Coriolis tank experiments, we estimate $\varepsilon \approx \mathscr{O}\left(10^{-2} \mathrm{~m}^{2} \mathrm{~s}^{-3}\right)$ from the PIV measurements, so that $L_{s} \approx 0.01 \mathrm{~m}$ and $v_{t} \sim 10^{-4} \mathrm{~m}^{2} \mathrm{~s}^{-1}$. Using as typical values for the velocity scale $U=0.1 \mathrm{~m} \mathrm{~s}^{-1}$ (cf. §III) and the vertical scale of the gravity current $h=0.05 \mathrm{~m}$ yields a normalized turbulent eddy viscosity of $v_{t} /(U h) \sim 0.02$. We also expect that at higher Richardson number, as in the ocean, the length scale will be determined by buoyancy since $L_{b}<L_{s}$,

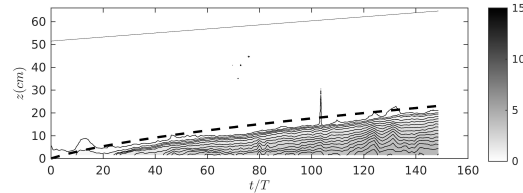

(a)

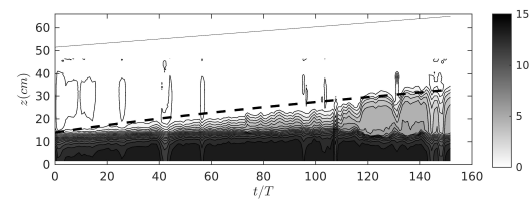

(c)

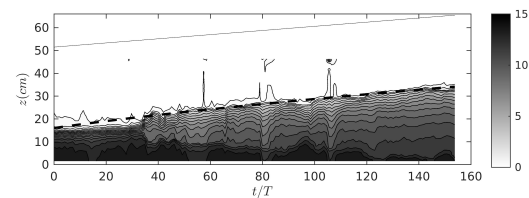

(e)

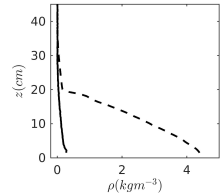

(b)

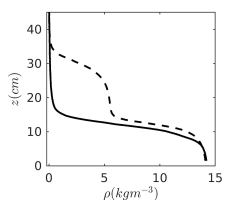

(d)

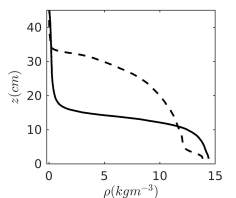

(f)
FIG. 8. Contour plots of the temporal evolution of the vertical density profile $(\mathrm{a}, \mathrm{c}, \mathrm{e})$ and selected vertical density profiles at $t / T=10$ (solid lines) and at $t / T=140$ (dashed lines) (b,d,f) in the central deep area for the experiment $H$ (top panels), $I 2$ (middle panels) and $B 1$ (bottom panels). The dashed line in (a,c,g) represents the theoretical increase of the pycnocline layer while the solid line gives the increase of the total water depth $H$ in the tank throughout the experiment.

$\left(L_{b}=\left(\varepsilon / N^{3}\right)^{1 / 2}\right.$ and $N$ the Brunt-Väisälä frequency) so that our oceanic estimate may be a bit high.

For parametrizations in ocean models Lane-Serff and Baines ${ }^{39}$ have used values in the range $0.03<v_{t}<$ $1.08 \mathrm{~m}^{2} \mathrm{~s}^{-1}$ for typical overflow scenarios. In the case of the Denmark Strait overflow between Iceland and Greenland and down into the Irminger Basin in the northern Atlantic (Dickson and Brown ${ }^{72}$ ) eddies have been observed by Krauss ${ }^{73}$ and Bruce ${ }^{74}$. Lane-Serff and Baines ${ }^{39}$ estimated for this case a vertical eddy diffusivity of $v_{t} \approx 1.08 \mathrm{~m}^{2} \mathrm{~s}^{-1}$, based on Dickson and Brown ${ }^{72}$ 's observational data. Using $U \sim 1 \mathrm{~ms}^{-1}$ as a typical velocity scale for the alongslope velocity in the Denmark Strait and a typical vertical length scale of the overflow of $h \sim 500 \mathrm{~m}$, it leads to a nondimensionalized turbulent viscosity of $v_{t} /(U h) \approx 0.002$ for this oceanographic scenario.

The values are smaller than the above presented estimates for the experimental tank due to weak shear and strong stratification combined with large Richardson numbers.

Hence, we expect the limit of the transition region of vortex formation to be shifted so that vortices develop for lower values of the viscous drainage $Y / L_{g}$.

The normalized vorticity $\zeta / f$ is plotted versus the stretching parameter $\Gamma$ in figure $7 \mathrm{~b}$. A lower stretching corresponds to a lower eddy rotation. Our data also fall within the previously reported values of Lane-Serff and Baines ${ }^{39}$. 


\section{GLOBAL CIRCULATION}

When the current reaches the central deep area or the layer of neutral buoyancy, an internal global circulation sets in, which is different depending on the depth considered, on the type of intrusion $(H, I, B)$ and the consequent evolution of the vertical density distribution of the receiving ambient, as well as on the rotation rate. Figure 8 shows the time evolution of a vertical density section taken from the conductivity probe at a distance from the centre of the tank of $r=108 \mathrm{~cm}$ (cf. figure 3). We show results relative to the experiments $H$ (a,b), $I 2$ (c,d) and $B 1$ (e,f) for simplicity, but results are similar for all other $I$ and $B$ experiments listed in table $I$ and have a general validity. In experiment $H$ the saline water reaches the bottom of the central deep area and fills the bottom layer according to the theoretical increase of the bottom depth $h_{b}$ obtained solving $d h_{b} / d t=Q /\left(\pi R^{2}(t)\right)$, where $R(t)$ is the radius at the depth reached at the time $t$ that increases from the bottom to the top of the slope $s$ as $R(t)=R_{0}+h_{b}(t) / s$, with $R_{0}=2.5 \mathrm{~m}$ being the radius of the central deep area at the bottom (see dashed line in figures $8(\mathrm{a}, \mathrm{c}, \mathrm{e})$ ). The stratification in the deepening bottom layer evolves in a nearly linear profile with a clear pycnocline as seen from figure $8(\mathrm{~b})$, where the solid line represent the initial stage at $t / T=5$ and the dashed line a later time at $t / T=140$.

In experiment $I 2$, the intrusion corresponding to $\rho_{j}$ is evident in figure 8(c) at $t / T \sim 10$. From figure $8(\mathrm{~d})$, it is evident that the initial two-layer stratification (solid line) in the central deep area evolves in a nearby three-layer ambient (dashed line), hence suggesting limited mixing with the initial twolayer ambient waters.

In experiment $B 1$, the intrusion happens more uniformly in the full bottom ambient layer, as seen in figure 8(e) where at $t / T=30$ we observe the thickening of the entire bottom layer: mixing is enhanced so that the pycnocline shifts at upper levels (cf. figures $8(\mathrm{e}, \mathrm{f})$ ). In the lower layer, the stratification becomes nearby parabolic. A dense thin layer at the bottom of the tank of roughly $4 \mathrm{~cm}$ thickness persists with the initial density $\rho_{b}$ that does not mix with the intrusion.

Figure 9 displays the time series of the mean curl averaged within the central deep area $\langle\zeta\rangle_{\phi, r<R_{0}}$ for the experiments $H$ (a), $I 2$ (b) and $B I$ (c) at a top level $z=45 \mathrm{~cm}$ (solid lines), an intermediate level $z=17 \mathrm{~cm}$ (dashed lines) and bottom level $z=7 \mathrm{~cm}$ (dotted lines). The vertical grey dashed lines represent the times at which the vertical density gradient from the conductivity probe registers a consequent intrusion (cf. figure 8).

In the experiment $H$, the top layers are characterized by an anticyclonic circulation as seen from the continuous line in figure 9a. At the top layers on the slope area, the developed baroclinic vortices generated by the gravity current induce in their interior part a ring of stronger anticyclonic circulation that smoothens moving toward the centre of the tank. This is highlighted by the instantaneous PIV velocity field at the first level $z=49 \mathrm{~cm}$ and at $t / T=88$ in figure $11 \mathrm{a}$, where arrows represent velocity vectors and the colorbar the norm of the velocity. 8 of the 9 baroclinic cyclones are evident, as well as the anticyclonic jet in the inner part of the vor-

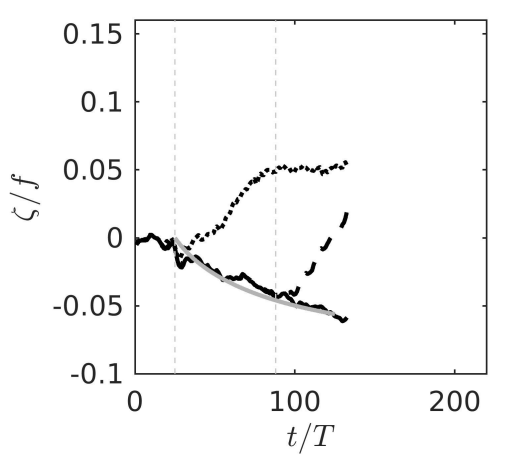

(a)

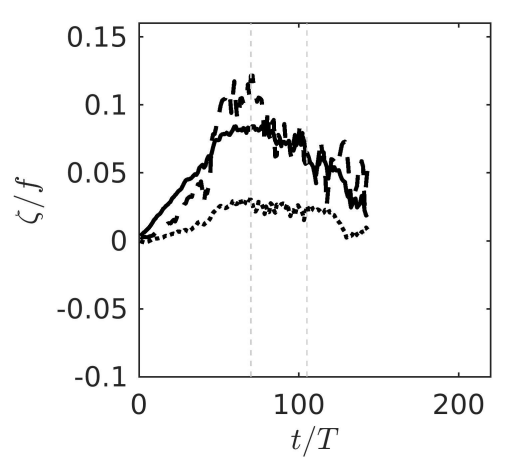

(b)

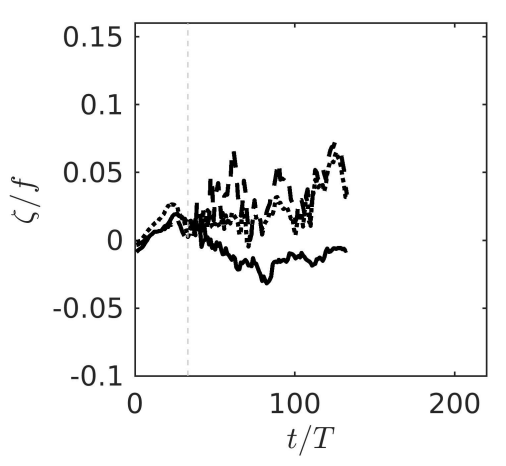

(c)

FIG. 9. Normalized averaged vorticity $\zeta / f$ in the central deep area $\left(r<R_{0}=250 \mathrm{~cm}\right)$ versus time for experiments $H$ (a), $I 2$ (b) and $B 1$ (c) with $T=90 \mathrm{~s}$ at the top layer (solid lines), at the intermediate layer (dashed lines) and the bottom layer (dotted lines). The vertical dashed grey lines are the instants at which the conductivity probe registers a conspicuous salt intrusion. The solid grey line in (a) gives equation 8

tices. At $t / T=50$ the level at $z=8 \mathrm{~cm}$ (dotted line in figure 9a) switches its circulation to cyclonic, because the bottom layer is rising (stretching) as the gravity current fills the bottom and the velocities are directed toward the centre of the tank. The same behavior is observed at later times $t / T=100$ when the saline bottom layer has reached the intermediate layer $z=17 \mathrm{~cm}$ (dashed line).

In the top fresh water ambient layer, there is an outward movement and a compression of the top layer because of ge- 


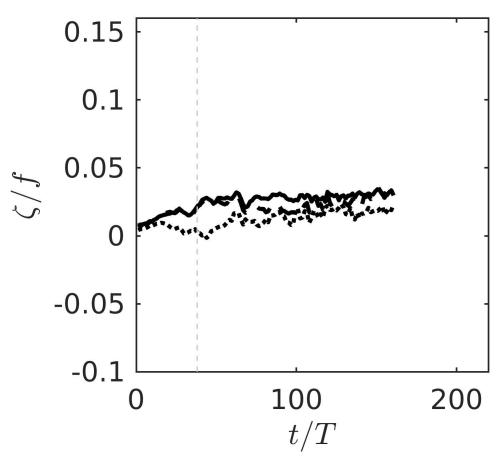

(a)

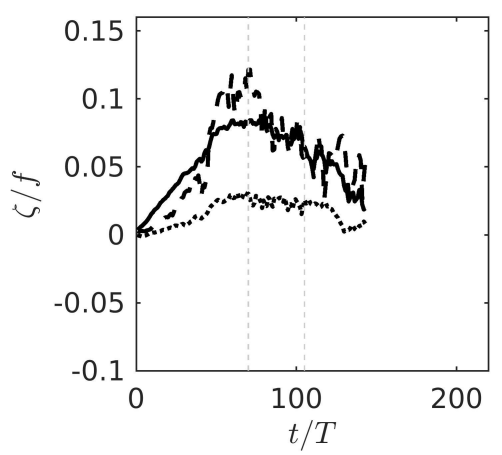

(d)

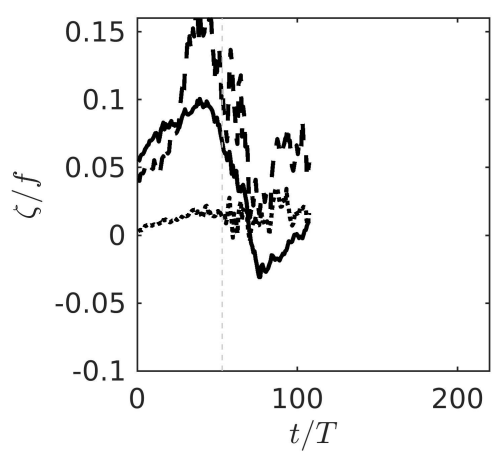

(g)

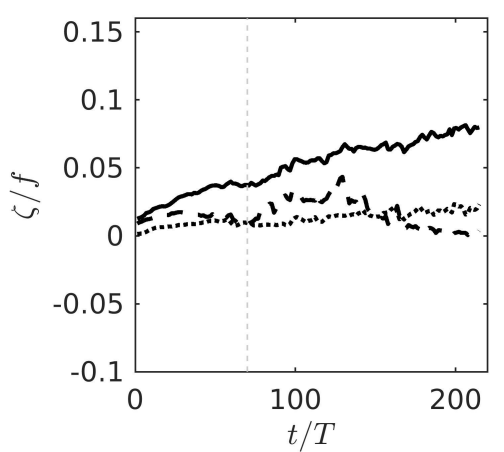

(b)

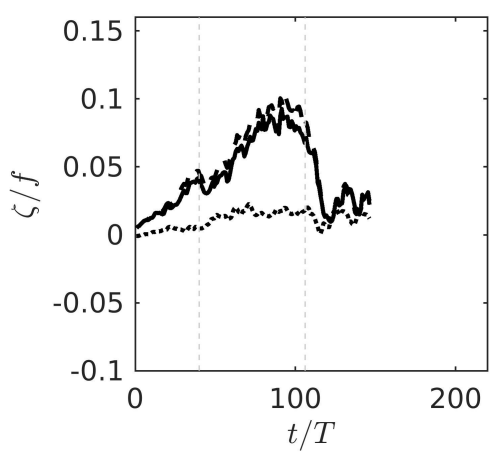

(e)

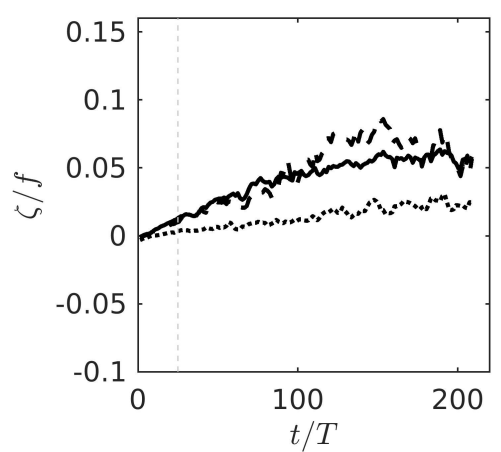

(c)

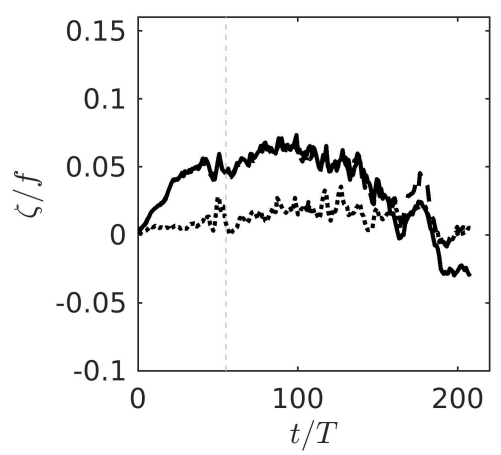

(f)

FIG. 10. Normalized averaged vorticity $\zeta / f$ in the central deep area $\left(r<R_{0}=250 \mathrm{~cm}\right)$ versus time for experiments $I 5$ (a),I4 (b), I6 (c) with $f=0.21 \mathrm{~s}^{-1}$, for experiments $I 2(\mathrm{~d}), I I(\mathrm{e}), I 3$ (f) with $f=0.14 \mathrm{~s}^{-1}$ and for experiments $I 6(\mathrm{~g})$ with $f=0.105 \mathrm{~s}^{-1}$. The vertical dashed grey lines are the instants at which the conductivity probe registers a conspicuous salt intrusion.

ometry. It is a combination of this compression and the consequent outward movement along with the ring of intense anticyclonic circulation induced by the baroclinic vortices that induces this anticyclonic circulation in the freshwater top layers. The solid grey line in figure 9a shows the theoretical vorticity decrease induced by the variation of the layer depth, assuming conservation of potential vorticity, i.e.

$$
\frac{d \zeta}{d t}=\frac{f}{h_{t}} \frac{d h_{t}}{d t} .
$$

As illustrated in figure 9a by the solid grey line, we see that this expression well predicts the anticyclonic circulation in the top layer.
A global view of the circulation in experiment $H$ is given in figure 12, where the azimuthal average of the along slope velocities $\langle v(r, z, t)\rangle_{\phi}$ is plotted in the $(r, z)$ plane at $t / T=70$ : we see that in the baroclinic vortices and along the slope the velocity is positive (cyclonic) while in the rest of the tank the circulation is anticyclonic, with the strong jet close to the inner part of the baroclinic vortices evident.

In the intrusive case $I$, shown in figure $9 \mathrm{~b}$, the scenario is quite different: the circulation remains always cyclonic in all layers with a monotonic increase during the first part of the experiment $t / T<70-90$ and a decrease in the last part of the experiment. On the slope area, the vein and Ekman layer turn cyclonically (cf. also radial velocity from the ADVP measurements in figure 4 ) and figure $12 \mathrm{~b}$. When the saline wa- 
ter on the slope reaches the pycnocline, it detaches from the boundary. Instead penetrating uniformly around the circumference toward the centre of the tank, it deviates and creates a ring of strong cyclonic circulation adjacent to the slope, a deep boundary current, where the saline water descending the slope accumulates and gives rise to localized bursts of strong intrusions often in form of dipoles that propagate toward the centre of the tank, as we observe from visualizations using dye injections [see Fig. 14 (Multimedia view)]. At the location of accumulation right adjacent to the slope, the flow in the upper and lower adjacent layers is pushed up/down, respectively, thus inducing a circulation as explained in detail in 66 . Hence, on the slope area above the current we observe a strong anticyclonic circulation enhanced by the circulation induced by the baroclinic surface vortices, while in the central deep area we have a global cyclonic circulation, which is strongest at the intermediate and top layers, but also weakly present in the bottom layers, below the intrusion (cf. figure 12b).

We note that the peak in the cyclonic circulation is reached approximately at the time at which the conductivity probe registers a conspicuous intrusion, as seen from the thickening of the density isolines corresponding to the intrusion density in figure $8 \mathrm{c}$ and highlighted by the vertical grey dashed lines in the figure. Since the intrusion is not uniform around the circumference and happens in localized bursts as explained above, there may be a time lap between the appearance of the peak in the vorticity plots and and the time at which the conductivity probe positioned at $r=108 \mathrm{~cm}$ registers the arrival of the saline intrusion.

In the bottom case, shown in figure $9 \mathrm{c}$, the compression of the bottom and top layers at the intrusion front is not present as in the intrusive case: since the intrusion is distributed over the entire bottom layer depth, the saline water moves inward and a cyclonic circulation is induced (see dashed and dotted lines in the figure), while the top layers are compressed moves outward and an anticyclonic circulation sets in quickly (cf. continuous line in figure 9c). Hence, the bottom intrusive case $B$ behaves similarly to the homogeneous case in concern of the global circulation.

It is interesting to look also at the influence of the background rotation. Figure 10 shows times series as in figure 9, but for intrusive experiments $I$ only (cf. table I) and with three different rotation rates with $f=0.21 \mathrm{~s}^{-1}$ (a,b,c), $f=0.14 \mathrm{~s}^{-1}$ $(\mathrm{d}, \mathrm{e}, \mathrm{f})$ and $f=0.105 \mathrm{~s}^{-1}(\mathrm{~g})$. In the strongest rotation cases (a,b,c), a monotonic increasing cyclonic circulation in all layers is reported, without observing a peak. In this case, the penetration is inhibited and the penetration distance is reduced (see Wirth and Negretti ${ }^{66}$ ), hence the circulation as depicted in figure 12 is sustained for longer times. As rotation decreases $(\mathrm{d}, \mathrm{e}, \mathrm{f}))$, the penetration distance increases and the local effect of upward and downward velocities close to the accumulation region adjacent to the slope is less remarked as in the stronger rotation case. Close to the end of the experiment at $t / T=140$ the circulation has become close to zero and even becomes anticyclonic for $t / T>180$ as given in figure 10(f). For experiment $I 4$ once the we observe first an increase and then a decrease of $\mathrm{C}$ circulation in all layers as

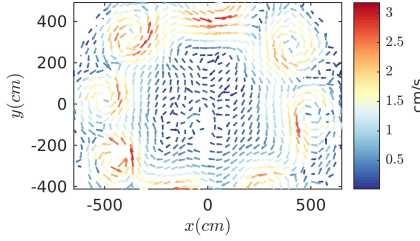

(a)

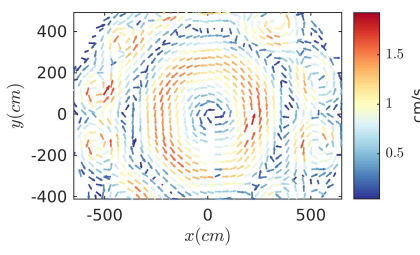

(c)

(e)

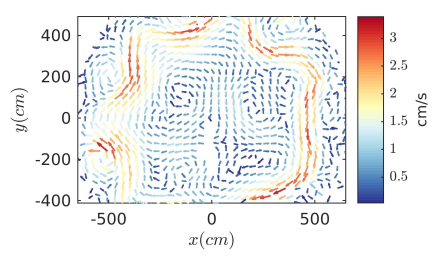

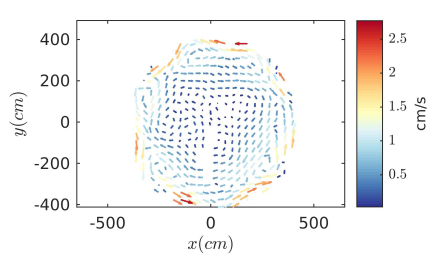

(b)

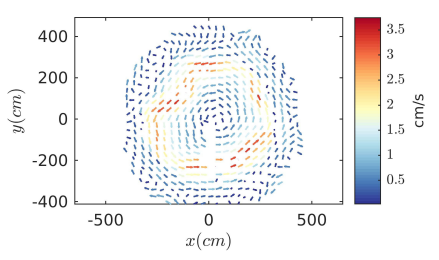

(d)

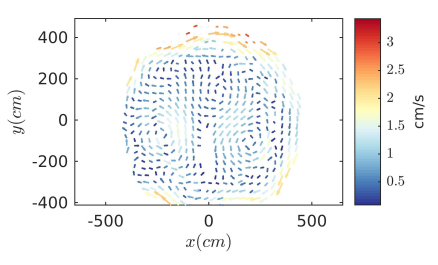

(f)
FIG. 11. Instantaneous velocity fields for experiment $H(\mathrm{a}, \mathrm{b})$, experiment $I 2(\mathrm{c}, \mathrm{d})$ and experiment $B I(\mathrm{e}, \mathrm{f})$ at the top layer $z=49 \mathrm{~cm}(\mathrm{a}, \mathrm{c}, \mathrm{e})$ and at the bottom $z=10 \mathrm{~cm}$ (b) and intermediate $(z=16 \mathrm{~cm})$ layers (d,f) respectively, at different times: (a) $t / T=68$, (b) $t / T=102$, (c) $t / T=102$, (d) $t / T=110$, (e) $t / T=115$, (f) $t / T=109$.

explained above. When the rotation is small $f=0.105 \mathrm{~s}^{-1}$, the penetration is facilitated and the penetration distance increases rapidly over the full pycnocline so that the $\mathrm{C}$ circulation ceases rapidly everywhere and eventually becomes $\mathrm{AC}$ in the top layers.

\section{PRODUCTION OF VORTICITY IN THE CENTRAL DEEP AREA}

After roughly $t / T \approx 20-40$ depending on the experiments $I$ or $B$, the gravity current has reached the level of neutral buoyancy, it detaches from the bottom boundary and penetrates in the central deep area with a strength that depends on the rotation rate. While experiment $H$ is characterized by the global circulation detailed above and reported in figure $12 \mathrm{a}$, in experiments $I$ and $B$, beside this overall circulation, a rich 


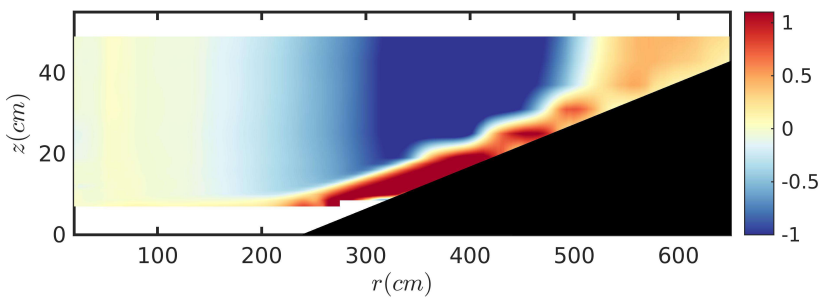

(a)

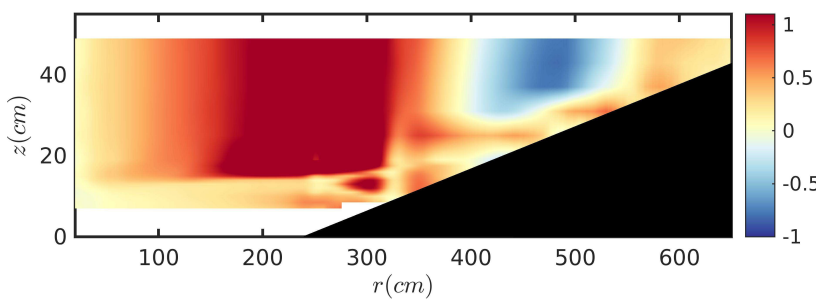

(b)

FIG. 12. Azimuthal average of the along slope velocity component $\left\langle v_{\theta}(r, z, t)\right\rangle_{\theta}$ in $\left[\mathrm{cms}^{-1}\right]$ at $t / T=70$ for experiment $H$ (a) and for experiment $I 2$ (b).

production of several localized cyclonic and anticyclonic vortices has been reported, that must be induced by the detaching saline intrusion, that is absent in experiment $H$, where the saline water follows the slope until it reaches the bottom of the central deep area. Typical scenarios for experiments $I$ and $B$ are displayed in figure 13, which represents a top view of dye visualizations in which Rhodamine $6 \mathrm{G}$ has been added to the saline injection and makes visible an intermediate level of experiment $B 1$ at $t / T=50$ (a) and at $t / T=140$ (b). In figure $13 \mathrm{a}$, the detaching boundary layer in form of shear instabilities are clearly evident with an ejection event in form of a dipole, which self propagates toward the central area by mutual advection of the counter-rotating vortices. This phenomenon has been also observed in the numerical studies of Vic et al. ${ }^{57}$ and Akuetevi and Wirth ${ }^{58}$, who found that submesoscale vortices are generated by the roll-up of frictional vortex filaments and engender dipoles that propagate balistically over distances several times their extension (see also Wenegrat, Callies, and Thomas ${ }^{59}$ ). After 90 rotational periods, the environment in the central deep area has changed significantly, with a rich ambient of cyclonic and anticyclonic submesoscales vortices of difference size, as seen in figure $13 b$.

Figure 15 displays the instantaneous vorticity fields for experiment $B l$ at $z=15 \mathrm{~cm}$ from the bottom at different times. At $t / T=5$ the vorticity is nearby zero everywhere. At a later time $t / T=55$, a localized injection event with opposite vorticity that grows and propagates cyclonically is evident in figure $15 \mathrm{~b}$ (cf. also figure 8). Clearly, filaments of vorticity and stretched vortical structures start to develop. They propagate toward the central area as seen from the vorticity field at $t / T=76$ (figure 15c) taking the form of coherent vortices that persist also at later times, $t / T=112$ (figure $15 \mathrm{~d}$ ), with further localized intrusion events still happening. A similar behaviour has been reported for all experiments conducted with

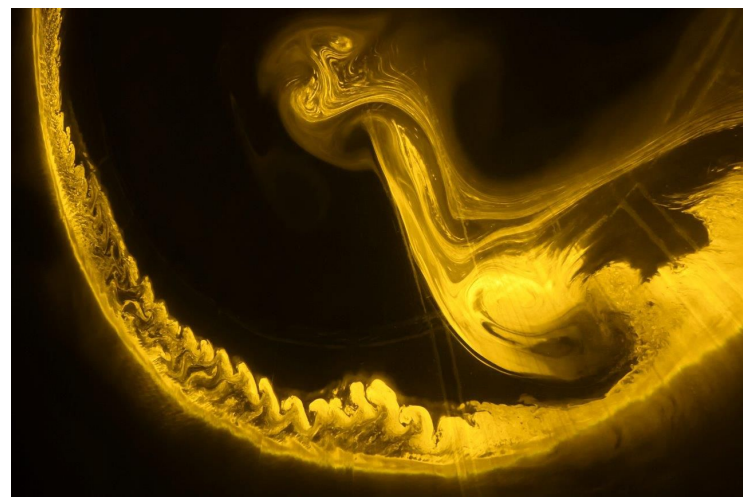

(a)

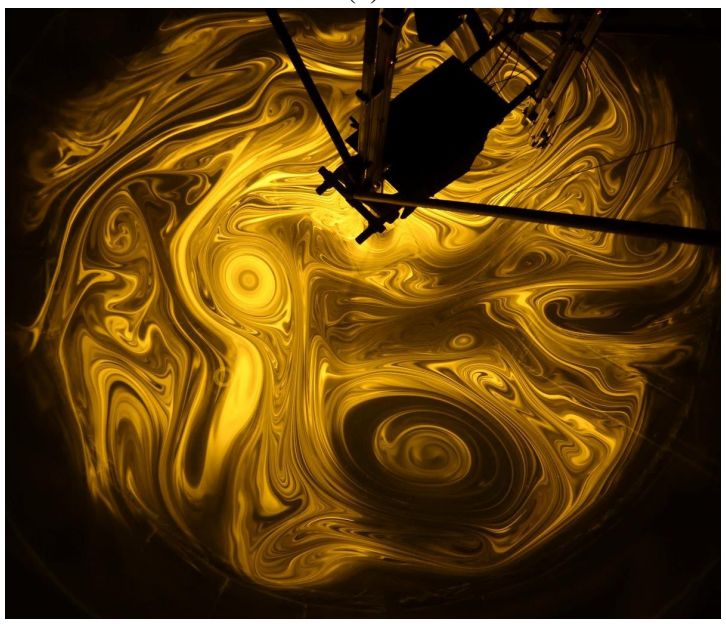

(b)

FIG. 13. Top view of an intermediate layer of experiment $B 1$ with the saline intrusion highlighted by fluorescent dye. (a) At $t / T=45$ the current starts intruding the ambient water in the central deep area often in form of dipole ejection. Also, the detaching frictional boundary layer is evident. (b) At $t / T=120$ the environment in the central deep area has clearly evolved with the presence of several localized coherent vortices both cyclonic and anticyclonic. The horizontal and vertical dimensions of the images are respectively $3 \times 2 \mathrm{~m}$ in (a) and $2.6 \times 2.6 \mathrm{~m}$ in $(\mathrm{b})$.

exception of the homogeneous case $H$. The distribution of the vorticity for experiment $H$ at a distance $z=12 \mathrm{~cm}$ from the bottom at different times is displayed in figure 16. Apart the areas of strong vorticity induced by the global circulation with main azimuthal velocity component that switches as seen in figure 12c, the central deep area do not exhibit at all the onset of vorticity patches throughout the experiment, even at later times when the intrusion has filled the bottom of the central deep area and produced a stratification in the ambient water (cf figure 8(a)).

The effect of the intrusion in creating coherent submesoscale vortices (i.e. with a typical size smaller the Rossby deformation radius) in the central deep area can be measured using the enstrophy $\zeta^{2}$ integrated over $0<R / R_{0}<1.3$ and $0<\theta<2 \pi$, where $R / R_{0}=1.3$ represents the approximate distance from the center of the tank where the saline injected water detaches from the slope boundary and intrudes. 


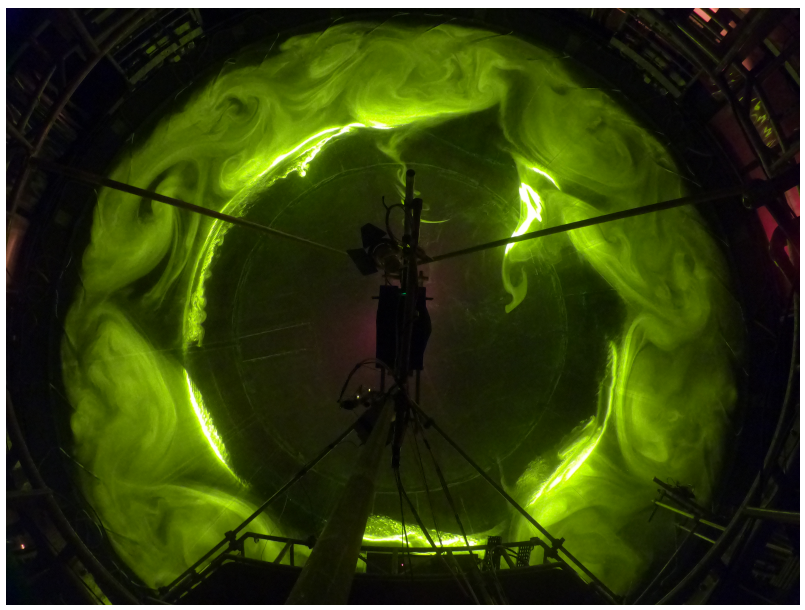

FIG. 14. Top view of experiment $I 2$ with the saline intrusion highlighted by fluorescent dye (Multimedia view).

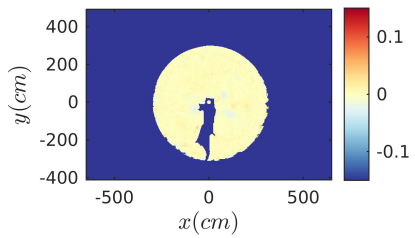

(a)

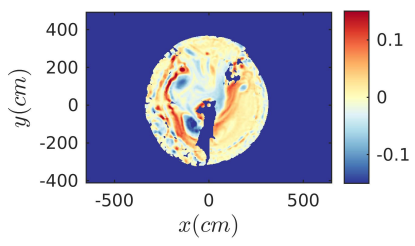

(c)

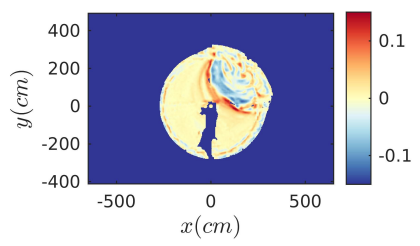

(b)

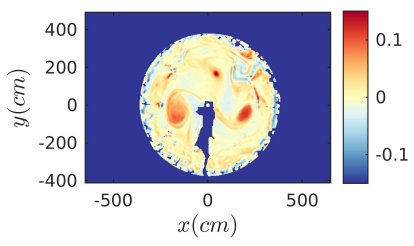

(d)
FIG. 15. Instantaneous vorticity field $\zeta(x, y, z, t)$ in $\left[\mathrm{s}^{-1}\right]$ at $z=15 \mathrm{~cm}$ and $t / T=5$ (a), $t / T=55$ (b), $t / T=76$ (c), $t / T=112$ (d) for experiment $B 1$.

Figure 17 shows the time evolution of the normalized enstrophy $\zeta^{2} / f^{2}$ for the three experiments $H, I 2$ and $B 1$ (b,d,f, respectively) at three different depths: one representative of the surface layers at $z=49 \mathrm{~cm}$ (continuous line), one at the intermediate level $z=16 \mathrm{~cm}$ (dashed line) and one at the bottom $z=8 \mathrm{~cm}$ (dotted line). For experiment $H$, the enstrophy remains almost constant throughout the experiment at each level, with exception of the small increment at a bottom level localized in time at $45<t / T<75$ that disappears after. The gravity current fills the bottom uniformly and without significant formation of localized coherent vortices in the intermediate levels, as mentioned above.

For experiment $I 2$, the enstrophy increases significantly from $t / T>45$ when the saline intrusion reaches the level of neutral buoyancy at the intermediate levels (solid line) and maintains at this level throughout the experimental duration.

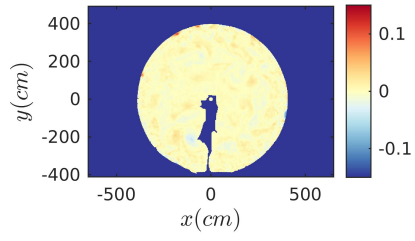

(a)

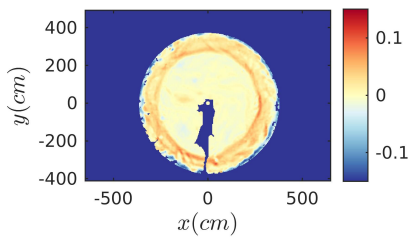

(c) (b)

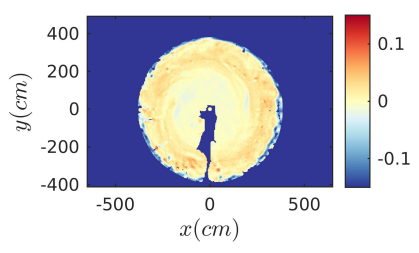

(d)

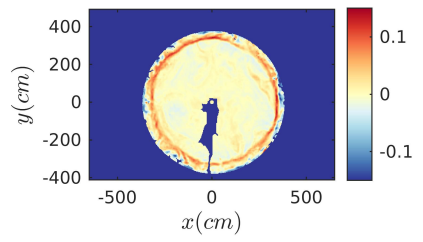

FIG. 16. Instantaneous vorticity field $\zeta(x, y, z, t)$ in $\left[\mathrm{s}^{-1}\right]$ at $z=12 \mathrm{~cm}$ at $t / T=5(\mathrm{a}), t / T=28$ (b), $t / T=68$ (c), $t / T=114$ (d) for experiment $H$.

The top and lower levels (dashed and dotted lines, respectively) keep a low constant enstrophy throughout the experiment. Considering the evolution of the vertical density profiles as given in figure $8(a, b, c)$, dissipative processes such as mixing are limited and need large time scales to become relevant so that the behaviour at the different levels (top, bottom, intermediate) remains different throughout the experiment.

For experiment $B 1$, the intrusion starts penetrating at $t / T=$ 20 at levels even above the initial pycnocline (cf. figure $8(\mathrm{~g}, \mathrm{~h}, \mathrm{i}))$ and a sudden increase of the enstrophy is reported at the intermediate levels, but also smoothly in both the bottom and top layers, even if with a smaller magnitude, of $\approx 1 / 3$ with respect to the intermediate levels. At $t / T=90$, the enstrophy in the intermediate levels ceases rapidly and the curves of all levels merge together keeping a constant magnitude with a smooth decrease in time. Here, a more intense mixing as given by the density vertical profile time evolution in figure $8(\mathrm{~g}, \mathrm{~h}, \mathrm{i})$ must take place so that enstrophy is dissipated quicker as compared to the intrusive case I2. Also, figure 17 suggests that the formation of submesoscale vortices can be better maintained for sharp two-layer density stratifications as in experiments $I$. We expect that for very long times, the scenarios of cases $I$ tend to approach those of the cases $B$ as mixing increases and the vertical density profile smoothens.

\section{SUMMARY AND CONCLUSIONS}

This article presents results from laboratory experiments of rotating intrusive gravity currents performed at the Coriolis Rotating Platform in Grenoble, France. An original experimental set-up has been employed using a uniform injection around the platform's circumference at the top of an inclined boundary, shaped as an inverted cone with a circular cross section descending toward the center of the tank. This configura- 


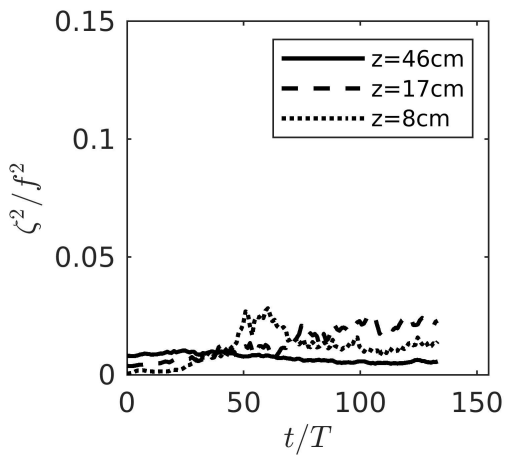

(a)

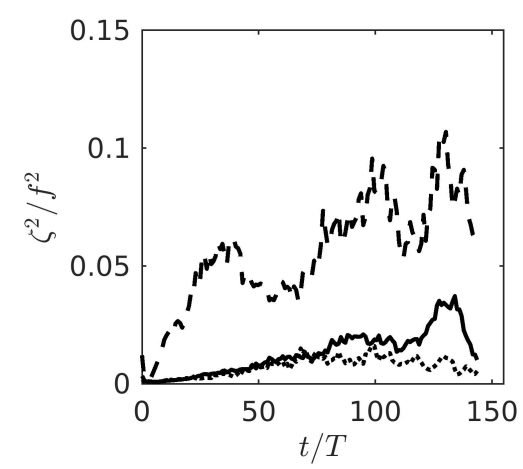

(b)

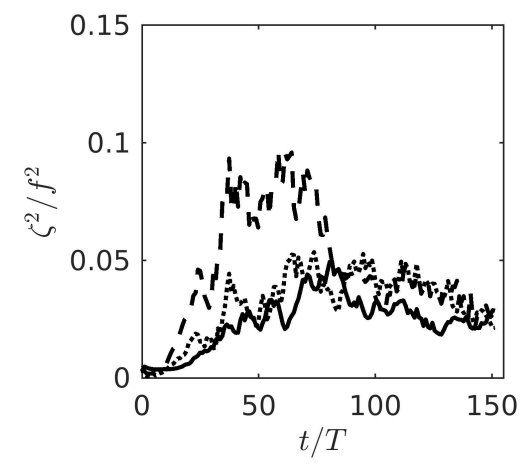

(c)

FIG. 17. Temporal evolution of the normalized enstrophy $\left\langle\zeta^{2}(t)\right\rangle_{\theta, r} / f^{2}$ averaged in the central deep area within $0<r / R_{0}<$ 1.1 at $z=49 \mathrm{~cm}$ (solid lines), $z=16 \mathrm{~cm}$ (dashed lines) and $z=8 \mathrm{~cm}$ (dotted lines) for the experiments $H$ (a), $I 2$ (b) and $B 1$ (c).

tion allowed to make azimuthal averages while monitoring the development of the gravity current on the slope, its intrusion in the central deep area and the evolution of the circulation in the central deep area for long experimental duration, up to 220 rotational periods. Velocity measurements were performed using Particle Image Velocimetry technique in the full tank in 16 horizontal levels at different vertical positions and using an acoustic velocimeter profiler to measure the velocities in the boundary layer in a vertical section of $3.2 \mathrm{~cm}$ on the slope spanning a radial section of $1 \mathrm{~m}$ length. The evolution of the stratification in the central deep area was monitored using a conductivity probe mounted on a vertical axe that moved between $z=1 \mathrm{~cm}$ and $z=51 \mathrm{~cm}$ from the bottom throughout the experiment. Data has been analyzed to characterize the gravity current itself and its scalings, the surface baroclinic vortices induced by the gravity current, and the global circulation and vorticity production induced in the central deep area by the intrusion.

As observed in previous studies $38,39,44,46,47$, the rotating gravity current produces cyclonic vortices in the surface layers. Their averaged characteristics such as their size, the propagation speed and their mean vorticity has been determined revealing a fair agreement with the previous observations $38,39,46,47$. In contrast, we report the formation of eddies in the parameter space of the normalized viscous drainage $Y / L_{g}$ and the stretching parameter $\Gamma$ defined by Lane-Serff and Baines ${ }^{38}$, where previous studies did not observe their generation. Considering the topographic $\beta$-plane, we report a correlation between both the observed wavenumber of the surface vortices and the topographic Rossby wavenumber, along with the related phase speeds.

The discrepancies observed between our results and those of previous studies are hence attributable to both the influence of topographic Rossby waves present in our study and viscous effects that are much reduced in the Coriolis Platform.

The variation parameters in the performed experiments were the rotation rate and the type of intrusion. In particular, we distinguished a homogeneous case $H$ consisting of a bottom gravity current, an intrusive case $I$ with the injection density being between the top and bottom two-layer stratified ambient densities, and a bottom intrusive case $B$ with the injection density very close to that of the bottom layer of the two-layer stratified ambient in the central deep area.

The global circulation induced by the gravity currents being intruding or following the slope until the bottom of the central deep area reveals to be very different.

Dense (bottom) gravity currents into a homogeneous ambient $H$ turns cyclonically along the slope and induce a global cyclonic circulation in the salty layer while filling the bottom of the central deep area, whereas the fresh ambient water layer turns anticyclonically because of the compression and consequent outward movement in the top layer.

Intrusive gravity currents $I$ with injection densities intermediate from the top and bottom two-layers ambient densities produce at the intrusion location an upward movement that creates a region of anticyclonic circulation on the slope area and a global cyclonic circulation in the central deep area, for all layers considered. As the intrusion propagates into the central deep area creating a third layer, the cyclonic circulation in the top and bottom layers decreases due to compression of the layers.

In the bottom intrusive cases $B$ with an injection density close to the initial bottom ambient layer density, the global circulation is more similar to the homogeneous case as the intrusion takes place in the entire bottom layer, hence stretching it, while compressing the top layer, inducing hence a cyclonic and an anticyclonic global circulation, respectively.

Intrusive $I$ and bottom intrusive $B$ cases revealed to produce strong turbulent environments in the central deep area at intermediate and bottom layers, in contrast to the dense cur- 
rent which follows the slope until the bottom of the central deep area, where the vorticity in the central deep area remains constant at low values throughout the experiment at all levels.

One important question that remains unanswered is whether submesoscale turbulence in the ocean interior can be created by frictional processes that occur in the boundary layer, with structures that detach from the bottom slope and form coherent vortices, as recently investigated in the studies of Molemaker, McWilliams, and Dewar ${ }^{56}$, Vic et al. ${ }^{57}$, Wenegrat, Callies, and Thomas ${ }^{59}$ and Gula, Molemaker, and McWilliams ${ }^{55}$. The detachment and propagation mechanisms, still unknown, are likely to be induced by baroclinic instabilities and take the form of dipoles, with their own propagation speed induced by mutual advection (Akuetevi and Wirth ${ }^{58}$ ). Frictional effects are present in both intrusive and bottom gravity currents. However, the main difference between these two cases is that in the former the boundary layer can detach and freely develop in the ambient water interior, carrying with it the small-scale turbulent structures present in the boundary layer. The size of these turbulent structures begins of the order of the Ekman layer thickness or smaller, and they may then evolve into larger structures, which we call 'submesoscales', that remain smaller than the Rossby deformation radius $(1 \mathrm{~m})$. In the bottom gravity current case, the current always remains attached to the bottom slope and fills the central deep area, so that in this case the small scale turbulent structures remain 'trapped' in the boundary layer and cannot freely develop in the ambient's interior. In this case, a global circulation is induced in the central deep area, but the vorticity remain low throughout the experiment in all layers.

These results suggest that the global circulation in the central deep area and in particular the formation of submesoscale vortices of different size and sign is closely related to boundary layer processes that can be transmitted into the ambient interior only in intrusive-type currents (experiments $I$ and $B$ ) and not in cases where the current follows the slope until the bottom of the central deep area as in experiment $H$, with important consequences on the mixing and re-stratification processes that take place in the ambient water.

\section{ACKNOWLEDGMENTS}

This work has been supported by a grant from SHOM, under contract number SHOM-20CP03. We are deeply indebted to S. Viboud and T. Valran for their support in conducting the experiments. Special thanks go to J. Sommeria for its support with the UVMAT software and for fruitful discussions.

\section{CONFLICT OF INTEREST}

The authors declare that they have no conflict of interest.

\section{DATA AVAILABILITY STATEMENT}

The data that support the findings of this study are available from the corresponding author upon reasonable request.

${ }^{1}$ J. Marschall and F. Schott, "Open-ocean convection: Observations, theory, and models," Rev. Geophys. 37(1), 1-64 (1999).

${ }^{2}$ J. Price, M. Barringer, R. Lueck, G. Johnson, I. Ambar, G. Parilla, A. Cantos, M. Kennely, and T. Sanford, "Abyssal upwelling and downwelling driven by near-boundary mixing," Science 259, 1277-1282 (1993).

${ }^{3}$ M. Hilt, L. Roblou, C. Nguyen, P. Marchesiello, F. Lemarié, S. Jullien, F. Dumas, L. Debreu, X. Capet, L. Bordois, R. Benshila, and F. Auclair, "Numerical modeling of hydraulic control, solitary waves and primary instabilities in the Strait of Gibraltar," Ocean Model. 155, 101642 (2020).

${ }^{4}$ M. Latif, E. Ozsoy, T. Oguz, and U. Unluata, "Observations of the mediterranean inflow into the black sea," Deep Sea Res. A 38, 711-23 (1991).

${ }^{5}$ A. Gordon, E. Zambianchi, A. Orsi, M. Visbeck, C. Giulivi, I. Whitworth, and G. Spezie, "Energetic plumes over the western ross sea continental slope." Geophys Res. Lett. 31, 21302 (2004).

${ }^{6}$ R. Dickson, E. Gmitrowicz, and A. Watson, "Deep-water renewal in the northern north atlantic," Nature 344, 848-850 (1990).

${ }^{7}$ G. Danabasoglu, W. Large, and B. Briegleb, J. Geophys. Res. 115, 1105 (2010).

${ }^{8}$ F. Auclair, L. Bordois, Y. Dossmann, T. Duhaut, A. Paci, C. Ulses, and C. Nguyen, "A non-hydrostatic non-boussinesq algorithm for free-surface ocean modelling," Ocean Model. 132, 12-29 (2018).

${ }^{9}$ M. Wells, C. Cenedese, and C. Caulfield, J. Phys. Ocean. 40, 2713-27 (2010).

${ }^{10}$ R. Ferrari and P. Wunsch, "Ocean circulation kinetic energy: Reservoirs, sources, and sinks," Ann Rev Fl Mech 41, 253-282 (2009).

${ }^{11}$ R. Ferrari, A. Mashayek, and T. e. a. McDougall, J. Phys. Ocean. 46(7), 2239-61 (2016).

${ }^{12}$ R. McDougall and R. Ferrari, "Abyssal upwelling and downwelling driven by near-boundary mixing,” J. Phys. Ocean. 47(2), 261-83 (2017).

${ }^{13}$ A. Rubino, M. Gačić, M. Bensi, V. Kovačević, V. Malačič, M. Menna, M. E. Negretti, J. Sommeria, D. Zanchettin, R. V. Barreto, L. Ursella, V. Cardin, G. Civitarese, M. Orlić, B. Petelin, and G. Siena, "Experimental evidence of long-term oceanic circulation reversals without wind influence in the north ionian sea." Sci. Rep. 10, 1905 (2020).

${ }^{14}$ J. S. Simpson, "Gravity currents in the laboratory, atmosphere and ocean," Ann. Rev. Fluid Mech. 14, 213-234 (1982).

${ }^{15}$ R. Britter and P. Linden, "The motion of the front of a gravity current travelling down an incline," J. Fluid Mech. 99, 531-543 (1980).

${ }^{16} \mathrm{C}$. Cenedese and C. Adduce, "A new parameterization for entrainment in overflows," J. Phys. Ocean. 40(8), 1835-50 (2010).

${ }^{17} \mathrm{M}$. Ungarish, An Introduction to Gravity Currents and Intrusions (CRC Press, 2009).

${ }^{18}$ J. Holyer and H. Huppert, "Gravity currents entering a two-layer fluid," J. Fluid Mech. 100, 739-767 (1980).

${ }^{19}$ H.-B. Cheong, J. J. P. Kuenen, and P. F. Linden, "The front speed of intrusive gravity currents," J. Fluid Mech. 552, 1-11 (2006).

${ }^{20}$ M. R. Flynn and P. F. Linden, "Intrusive gravity currents," J. Fluid Mech. 568, 193-202 (2006).

${ }^{21}$ B. R. Sutherland and J. T. Nault, "Intrusive gravity currents propagating along thin and thick interfaces," J. Fluid Mech. 586, 109-118 (2007).

${ }^{22}$ M. R. Flynn, M. Ungarish, and A. W. Tan, "Gravity currents in a twolayer stratified ambient: The theory for the steady-state (front condition) and lock-released flows, and experimental confirmations," Phys. Fluids 24, 026601 (2012).

${ }^{23}$ A. Dai, Y.-L. Huang, and Y.-M. Hsieh, "Gravity currents propagating at the base of a linearly stratified ambient," Phys. Fluids 33, 066601 (2021).

${ }^{24}$ L. Ottolenghi, C. Adduce, F. Roman, and G. la Forgia, "Large eddy simulations of solitons colliding with intrusions," Physics of Fluids 32, 096606 (2020).

${ }^{25}$ G. la Forgia, L. Ottolenghi, C. Adduce, and F. Falcini, "Intrusions and solitons: Propagation and collision dynamics," Physics of Fluids 32, 076605 (2020).

${ }^{26}$ T. H. Ellison and J. S. Turner, "Turbulent entrainment in stratified flows," J. Fluid Mech. 6, 423-448 (1959). 
${ }^{27}$ M. E. Negretti, J. B. Flòr, and E. J. Hopfinger, "Development of gravity currents on rapidly changing slopes," J. Fluid Mech. 833, 70-97 (2017).

${ }^{28}$ R. Kostaschuk, M. M. Nasr-Azadani, E. Meiburg, T. Wei, Z. Chen, M. E. Negretti, J. Best, J. Peakall, and D. Parsons, "On the causes of pulsing in continuous turbidity currents," Journal of Geophysical Research: Earth Surface 123, 2827-2843 (2018).

${ }^{29}$ A. Martin, M. E. Negretti, and E. J. Hopfinger, "Development of gravity currents on slopes under different interfacial instability conditions," J. Fluid Mech. 880, 180-208 (2019).

${ }^{30}$ T. Zemach, M. Ungarish, A. Martin, and M. E. Negretti, "On gravity currents of fixed volume that encounter a down-slope or up-slope bottom," Physics of Fluids 31, 096604 (2019).

${ }^{31}$ A. Martin, M. E. Negretti, M. Ungarish, and T. Zemach, "Propagation of a continuously supplied gravity current head down bottom slopes," Phys. Rev. Fluids 5, 054801 (2020).

${ }^{32}$ M. De Falco, C. Adduce, M. Negretti, and E. Hopfinger, "On the dynamics of quasi-steady gravity currents flowing up a slope," Advances in Water Resources 147, 103791 (2021).

${ }^{33} \mathrm{~A}$. Wirth, "On the basic structure of oceanic gravity currents," Ocean Dynamics 59, 551-563 (2009).

${ }^{34}$ R. Griffiths, "Gravity currents in rotating systems," Ann. Rev. Fl. Mech. 18, 59-89 (1986).

${ }^{35}$ L. Jiang and R. Garwood, "Three-dimensional simulations of overflows on continental slopes," J. Phys. Ocean. 26(7), 1214-33 (1996).

${ }^{36} \mathrm{~J}$. Jungclaus and J. Backhaus, "Application of a transient reduced gravity plume model to the denmark strait overflow," J. Geophys. Res. Oceans 99C6, 12375-96 (1994)

${ }^{37}$ G. Shapiro and A. Hill, "Dynamics of dense water cascades at the shelf edge," J. Phys. Ocean. 27, 2381-2394 (1997).

${ }^{38}$ G. F. Lane-Serff and P. G. Baines, "Eddy formation by dense flows on slopes in a rotating fluid," J. Fluid Mech. 363, 229-252 (1998).

${ }^{39}$ G. F. Lane-Serff and P. G. Baines, "Eddy formation by overflows in stratified water," J. Phys. Ocean. 30, 327-337 (2000).

${ }^{40} \mathrm{P}$. Choboter and G. Swaters, "On the baroclinic instability of axisymmetric rotating gravity currents with bottom slope," J. Fluid. Mech. 408, 149-77 (2000).

${ }^{41}$ G. I. Shapiro, J. M. Huthnance, and V. V. Ivanov, "Dense water cascading off the continental shelf," Journal of Geophysical Research: Oceans 108 (2003).

${ }^{42} \mathrm{C}$. Cenedese and C. Adduce, "Mixing in a density driven current down a slope in a rotating fluid," J. Fluid Mech. 604, 369-88 (2008).

${ }^{43}$ M. C. De Falco, C. Adduce, A. Cuthbertson, M. E. Negretti, J. Laanearu, D. Malcangio, and J. Sommeria, "Experimental study of uni- and bi-directional exchange flows in a large-scale rotating trapezoidal channel," Physics of Fluids 33, 036602 (2021).

${ }^{44} \mathrm{C}$. Cenedese, J. Whitehead, T. Ascarelli, and M. Ohiwa, "A dense current flowing down a sloping bottom in a rotating fluid," J. Phys. Ocean. 34, 188203 (2004).

${ }^{45}$ J. Whitehead, "Rotating gravity currents," Reference Module in Earth Systems and Environmental Sciences (2016).

${ }^{46}$ D. Etling, F. Gelhardt, U. Schrader, F. Brennecke, G. Kühn, G. d'Hieres, and H. Didelle, "Experiments with density currents on a sloping bottom in a rotating fluid," Dynamics of Atmospheres and Oceans 31, 0-164 (2000).

${ }^{47}$ S. Decamp and J. Sommeria, "Scaling properties for turbulent gravity currents deviated by coriolis effects on a uniform slope," (2009), unpublished.

${ }^{48}$ M. Spall and J. Price, "Mesoscale variability in denmark strait: the pv outflow hypothesis," J. Phys. Ocean. 28, 1598-623 (1998).

${ }^{49} \mathrm{D}$. Nof, "The translation of isolated cold eddies on a sloping bottom," Deep Sea Res. A 30, 171-82 (1983).

${ }^{50} \mathrm{~K}$. Speer, E. Tzipperman, and Y. Feliks, "Topography andgrounding in a simple layer model,” J. Geophys. Res. Oceans 98, 8547-58 (1993).
${ }^{51}$ B. Sutherland, J. Nault, K. Yewchuk, and G. Swaters, "A new parameterization for entrainment in overflows," J. Fluid Mech. 508, 241-64 (2004).

${ }^{52}$ E. Yankovsky and S. Legg, "Symmetric and baroclinic instability in dense shelf overflows," J. Phys. Ocean. 49, 39 (2019).

${ }^{53}$ R. W. Griffiths and E. J. Hopfinger, "Gravity currents moving along a lateral boundary in a rotating fluid," J. Fluid Mech. 134, 357-399 (1983).

${ }^{54} \mathrm{M}$. Spall, "Dynamics of downwelling in an eddy-resolving convective basin," J. Phys. Ocean. 10, 2341 (2010).

${ }^{55}$ J. Gula, M. J. Molemaker, and J. C. McWilliams, "Topographic generation of submesoscale centrifugal instability and energy dissipation," Nat. Commun. 7, 12811 (2016).

${ }^{56}$ M. Molemaker, J. McWilliams, and W. Dewar, "Submesoscale instability and generation of mesoscale anticy- clones near a separation of the california undercurrent." J. Phys. Ocean. 45, 613 (2015).

${ }^{57}$ C. Vic, G. Roullet, X. Capet, X. Carton, M. Molemaker, and J. Gula, "Eddy-topography interactions and the fate of the persian gulf outflow," J. Geophys. Res. Oceans 120, 6700-17 (2016).

${ }^{58} \mathrm{C}$. Akuetevi and A. Wirth, "Dynamics of turbulent western-boundary currents at low latitude in a shallow-water model." Ocean Sci. 11, 471-481 (2015).

${ }^{59}$ J. Wenegrat, J. Callies, and L. Thomas, "Submesoscale baroclinic instability in the bottom boundary layer," J. Phys. Ocean. 48, 2571-92 (2018).

${ }^{60} \mathrm{~S}$. Legg, R. W. Hallberg, and J. Girton, "Comparison of entrainment in overflows simulated by z-coordinate, isopycnal and non-hydrostatic models," Ocean Modell. 11, 69-97 (2006).

${ }^{61}$ E. C. Itsweire, K. N. Helland, and C. W. Van Atta, "The evolution of gridgenerated turbulence in a stably stratified fluid," J. Fluid Mech. 162, 299388 (1986).

${ }^{62}$ Y. Kyunghwan and Z. Warhaft, "The evolution of grid-generated turbulence under conditions of stable thermal stratification," J. Fluid Mech. 215, 60138 (1990).

${ }^{63}$ O. Praud, A. Fincham, and J. Sommeria, "Decaying grid turbulence in a strongly stratified fluid," J. Fluid. Mech. 522, 1-33 (2005).

${ }^{64}$ P. Augier, P. Billant, M. Negretti, and J.-M. Chomaz, "Experimental study of stratified turbulence forced with columnar dipoles," Physics of Fluids 26, 046603 (2014).

${ }^{65} \mathrm{Http} / / /$ www.legi.grenoble-inp.fr/web/spip.php?article1441.

${ }^{66}$ A. Wirth and M.-E. Negretti, "The circulation of (non) intruding gravity currents and their re-circulation in a rotating frame," Ocean Model. Submitted.

${ }^{67} \mathrm{~K}$. Hedstrom and L. Armi, "An experimental study of homogeneous lenses in a stratified rotating fluid," J. Fluid Mech. 191, 535-556 (1988).

${ }^{68}$ G. E. Swaters, "Numerical simulations of the baroclinic dynamics of density-driven coupled fronts and eddies on a sloping bottom," J. Geophys. Res. Oceans 103, 2945-61 (1998).

${ }^{69}$ M. Mory, M. Stern, and R. Griffiths, "Coherent baroclinic eddies on a sloping bottom," J. Fluid. Mech. 183, 45-62 (1987).

${ }^{70}$ A. Muraro, Dynamics and structure of coherent eddies generated by gravity currents, Master's thesis, LEGI, CNRS, Grenoble, France (2021), dir.: A. Wirth and M.E. Negretti, LEGI.

${ }^{71}$ C. J., P. Odier, and R. E. Ecke, "Understanding and modeling turbulent fluxes and entrainment in a gravity current,” Phys. D: Nonlin. Phen. (2012), 10.1016/j.physd.2011.07.010.

${ }^{72}$ R. Dickson and J. Brown, "The production of north atlantic deep water: Sources, rates and pathways," J. Geophys. Res. 99, 12319-41 (1994).

${ }^{73}$ W. Krauss, "A note on overflow eddies," Deep Sea Res. I 43, 1661-67 (1996).

${ }^{74}$ J. Bruce, "Eddies southwest of the denmark strait," Deep Sea Res. I 42, 13-29 (1995). 